\title{
Charged Higgs boson mass of the MSSM in the Feynman diagrammatic approach
}

\author{
M. Frank, ${ }^{1, *}$ L. Galeta, ${ }^{2, \dagger}$ T. Hahn, ${ }^{3, \sharp}$ S. Heinemeyer, ${ }^{2, \S}$ W. Hollik, ${ }^{3, \|}$ H. Rzehak, ${ }^{4, \mathbb{I}, * *}$ and G. Weiglein ${ }^{5, \dagger \dagger}$ \\ ${ }^{1}$ Institut für Theoretische Physik, Universität Karlsruhe, D-76128 Karlsruhe, Germany ${ }^{\ddagger}$ \\ ${ }^{2}$ Instituto de Física de Cantabria (CSIC-UC), Santander, Spain \\ ${ }^{3}$ Max-Planck-Institut für Physik (Werner-Heisenberg-Institut), Föhringer Ring 6, D-80805 München, Germany \\ ${ }^{4}$ CERN, PH-TH, 1211 Geneva 23, Switzerland \\ ${ }^{5}$ DESY, Notkestraße 85, D-22607 Hamburg, Germany \\ (Received 20 June 2013; published 16 September 2013)
}

\begin{abstract}
The interpretation of the Higgs signal at $\sim 126 \mathrm{GeV}$ within the Minimal Supersymmetric Standard Model (MSSM) depends crucially on the predicted properties of the other Higgs states of the model, as the mass of the charged Higgs boson, $M_{H^{ \pm}}$. This mass is calculated in the Feynman diagrammatic approach within the MSSM with real parameters. The result includes the complete one-loop contributions and the two-loop contributions of $\mathcal{O}\left(\alpha_{t} \alpha_{s}\right)$. The one-loop contributions lead to sizable shifts in the $M_{H^{ \pm}}$ prediction, reaching up to $\sim 8 \mathrm{GeV}$ for relatively small values of $M_{A}$. Even larger effects can occur depending on the sign and size of the $\mu$ parameter that enters the corrections affecting the relation between the bottom-quark mass and the bottom Yukawa coupling. The two-loop $\mathcal{O}\left(\alpha_{t} \alpha_{s}\right)$ terms can shift $M_{H^{ \pm}}$by more than $2 \mathrm{GeV}$. The two-loop contributions amount to typically about $30 \%$ of the one-loop corrections for the examples that we have studied. These effects can be relevant for precision analyses of the charged MSSM Higgs boson.
\end{abstract}

DOI: 10.1103/PhysRevD.88.055013

\section{INTRODUCTION}

The ATLAS and CMS experiments at CERN have recently discovered a new boson with a mass around $126 \mathrm{GeV}[1,2]$. Within the presently still rather large experimental uncertainties this new boson behaves like the Higgs boson of the Standard Model (SM) [3]. However, the newly discovered particle can also be interpreted as the Higgs boson of extended models. The Higgs sector of the Minimal Supersymmetric Standard Model (MSSM) [4] with two scalar doublets accommodates five physical Higgs bosons. In lowest order these are the light and heavy $C P$-even $h$ and $H$, the $C P$-odd $A$, and the charged Higgs bosons $H^{ \pm}$. It was shown that the newly discovered boson can be interpreted in principle as the light, but also as the heavy $C P$-even Higgs boson of the MSSM, see, e.g., Refs. [5-9]. In the latter case the charged Higgs boson must be rather light, and the search for the charged Higgs boson could be crucial to investigate this scenario [8]. In the former case the charged Higgs boson is bound to be heavier than the top quark [8]. In both cases the discovery of a charged Higgs boson would constitute an unambiguous sign of physics beyond the SM, serving as a good motivation for searches for the charged Higgs boson.

\footnotetext{
*m@rkusfrank.de

${ }^{\dagger}$ leo@ifca.unican.es

*hahn@feynarts.de

${ }_{\text {\$Sven.Heinemeyer@cern.ch }}$

"hollik@mppmu.mpg.de

Thrzehak@mail.cern.ch

**On leave from Albert-Ludwigs-Universität Freiburg,

Physikalisches Institut, D-79104 Freiburg, Germany.

Georg.Weiglein@desy.de

${ }^{*}$ Former address.
}

PACS numbers: 12.60.Fr, 12.60.Jv, 14.80.Da, 14.80.Ly

The Higgs sector of the MSSM can be expressed at lowest order in terms of the gauge couplings, the mass of the $C P$-odd Higgs boson, $M_{A}$, and $\tan \beta \equiv v_{2} / v_{1}$, the ratio of the two vacuum expectation values. All other masses and mixing angles can therefore be predicted, e.g. the charged Higgs boson mass,

$$
m_{H^{ \pm}}^{2}=M_{A}^{2}+M_{W}^{2},
$$

at tree level. $M_{Z, W}$ denote the masses of the $Z$ and $W$ boson, respectively. Higher-order contributions can give large corrections to the tree-level relations, where the loop corrected charged Higgs boson mass is denoted as $M_{H^{ \pm}}$.

Experimental searches for the neutral MSSM Higgs bosons have been performed at LEP [10,11], placing important restrictions on the parameter space. At Run II of the Tevatron the search was continued, but is now superseded by the LHC Higgs searches. Besides the discovery of a SM Higgs-like boson the LHC searches place stringent bounds, in particular in the regions of small $M_{A}$ and large $\tan \beta$ [12]. At a future linear collider (LC) a precise determination of the Higgs boson properties (either of the light Higgs boson at $\sim 126 \mathrm{GeV}$ or heavier MSSM Higgs bosons within the kinematic reach) will be possible [13-16]. The interplay of the LHC and the LC in the neutral MSSM Higgs sector has been discussed in Refs. [17,18].

The charged Higgs bosons of the MSSM (or a more general two Higgs doublet model) have also been searched for at LEP [19-23], yielding a bound of $M_{H^{ \pm}} \gtrsim 80 \mathrm{GeV}$ [24,25]. The LHC places bounds on the charged Higgs mass, as for the neutral heavy MSSM Higgs bosons, at relatively low values of its mass and at large or very small $\tan \beta$ [26,27]. For $m_{H^{ \pm}}<m_{t}$ (with $m_{t}$ denoting the mass of the top quark) the charged Higgs boson is mainly produced 
from top quarks and decays mainly as $H^{ \pm} \rightarrow \tau \nu_{\tau}$. For $m_{H^{ \pm}}>m_{t}$ the charged Higgs boson is mainly produced together with a top quark and the dominant decay channels are $H^{ \pm} \rightarrow t b, \tau \nu_{\tau}$, where the latter is the main search channel. At the LC, for $M_{H^{ \pm}} \lesssim \sqrt{s} / 2$ a high-precision determination of the charged Higgs boson properties will be possible [13-16].

For the MSSM $^{1}$ the status of higher-order corrections to the masses and mixing angles in the neutral Higgs sector is quite advanced. The complete one-loop result within the MSSM is known [30-33]. The by far dominant one-loop contribution is the $\mathcal{O}\left(\alpha_{t}\right)$ term due to top and stop loops [ $\alpha_{t} \equiv h_{t}^{2} /(4 \pi), h_{t}$ being the top-quark Yukawa coupling]. The computation of the two-loop corrections has meanwhile reached a stage where all the presumably dominant contributions are available [34-48]. In particular, the $\mathcal{O}\left(\alpha_{t} \alpha_{s}\right), \mathcal{O}\left(\alpha_{t}^{2}\right), \mathcal{O}\left(\alpha_{b} \alpha_{s}\right), \mathcal{O}\left(\alpha_{t} \alpha_{b}\right)$ and $\mathcal{O}\left(\alpha_{b}^{2}\right)$ contributions to the self-energies are known for vanishing external momenta. For the (s)bottom corrections, which are mainly relevant for large values of $\tan \beta$, an all-order resummation of the $\tan \beta$-enhanced term of $\mathcal{O}\left(\alpha_{b}\left(\alpha_{s} \tan \beta\right)^{n}\right)$ is performed [49-51]. The remaining theoretical uncertainty on the lightest $C P$-even Higgs boson mass has been estimated to be about $\sim 3 \mathrm{GeV}$ [52-54]. The public codes FeynHiggs $[28,35,52,55]$ (including all of the above corrections) and CPsuperH [56] exist. A full two-loop effective potential calculation (including even the momentum dependence for the leading pieces and the leading threeloop corrections) has been published [57]. However, no computer code is publicly available. Most recently another leading three-loop calculation, depending on the various supersymmetry (SUSY) mass hierarchies, became available [58], resulting in the code $\mathrm{H} 3 \mathrm{~m}$ (which adds the threeloop corrections to the FeynHiggs result).

Also the mass of the charged Higgs boson is affected by higher-order corrections. However, the status is somewhat less advanced as compared to the neutral Higgs bosons. First, in Ref. [59] leading corrections to the relation given in Eq. (1) have been evaluated. The one-loop corrections from $t / b$ and $\tilde{t} / \tilde{b}$ loops have been derived in Refs. [60,61]. A nearly complete one-loop calculation, neglecting the terms suppressed by higher powers of the SUSY mass scale, was presented in Ref. [62]. The first full one-loop calculation in the Feynman diagrammatic (FD) approach has been performed in Ref. [63], and reevaluated more recently in Refs. [28,64]. At the two-loop level, within the FD approach, the leading $\mathcal{O}\left(\alpha_{t} \alpha_{s}\right)$ two-loop contributions for the three neutral Higgs bosons in the case of complex soft SUSY-breaking parameters have been obtained [29]. Because of the ( $C P$-violating) mixing between all three neutral Higgs bosons, in the MSSM with complex parameters usually the charged Higgs mass is chosen as independent (on-shell) input parameter, which by construction does not receive any higher-order corrections. The calculation however involves the evaluation of the $\mathcal{O}\left(\alpha_{t} \alpha_{s}\right)$ contributions to the charged $H^{ \pm}$self-energy. In the $C P$-conserving case, on the other hand, where usually $M_{A}$ instead of $M_{H^{ \pm}}$is chosen as independent input parameter, the corresponding self-energy contribution can be utilized to obtain corrections of $\mathcal{O}\left(\alpha_{t} \alpha_{s}\right)$ to the mass $M_{H^{ \pm}}$.

In the present paper we combine the two-loop terms of $\mathcal{O}\left(\alpha_{t} \alpha_{s}\right)$ with the complete one-loop contribution of Ref. [28] to obtain an improved prediction for the mass of the charged Higgs boson. The results are incorporated in the code FeynHiggs (current version: 2.9.4). An overview of the calculation is given in Sec. II, whereas in Sec. III and IV we discuss the size and relevance of the one- and two-loop corrections and investigate the impact of the various sectors of the MSSM on the prediction for $M_{H^{ \pm}}$. Our conclusions are given in Sec. V.

\section{HIGHER-ORDER CONTRIBUTIONS FOR $M_{H^{ \pm}}$}

\section{A. From tree level to higher orders}

In the MSSM (with real parameters) one conventionally chooses the mass of the $C P$-odd Higgs boson, $M_{A}$, and $\tan \beta\left[\equiv v_{2} / v_{1}\right.$, see Eq. (2)] as independent input parameters. Thus the mass of the charged Higgs boson can be predicted in terms of the other parameters and receives a shift from the higher-order contributions.

The two Higgs doublets of the MSSM are decomposed in the following way,

$$
\begin{aligned}
& \mathcal{H}_{1}=\left(\begin{array}{l}
H_{11} \\
H_{12}
\end{array}\right)=\left(\begin{array}{c}
v_{1}+\frac{1}{\sqrt{2}}\left(\phi_{1}-i \chi_{1}\right) \\
-\phi_{1}^{-}
\end{array}\right), \\
& \mathcal{H}_{2}=\left(\begin{array}{l}
H_{21} \\
H_{22}
\end{array}\right)=\left(\begin{array}{c}
\phi_{2}^{+} \\
v_{2}+\frac{1}{\sqrt{2}}\left(\phi_{2}+i \chi_{2}\right)
\end{array}\right),
\end{aligned}
$$

with the two vacuum expectation values $v_{1}$ and $v_{2}$. The Hermitian $2 \times 2$ matrix of the charged states $\phi_{1,2}^{ \pm}, \mathbf{M}_{\phi^{ \pm} \phi^{ \pm}}$, contains the following elements:

$$
\mathbf{M}_{\phi^{ \pm} \phi^{ \pm}}=\left(\begin{array}{cc}
m_{1}^{2}+\frac{1}{4} g_{1}^{2}\left(\boldsymbol{v}_{1}^{2}-\boldsymbol{v}_{2}^{2}\right)+\frac{1}{4} g_{2}^{2}\left(\boldsymbol{v}_{1}^{2}+\boldsymbol{v}_{2}^{2}\right) & -m_{12}^{2}-\frac{1}{2} g_{2}^{2} \boldsymbol{v}_{1} \boldsymbol{v}_{2} \\
-m_{12}^{2}-\frac{1}{2} g_{2}^{2} \boldsymbol{v}_{1} \boldsymbol{v}_{2} & m_{2}^{2}+\frac{1}{4} g_{1}^{2}\left(\boldsymbol{v}_{2}^{2}-v_{1}^{2}\right)+\frac{1}{4} g_{2}^{2}\left(\boldsymbol{v}_{1}^{2}+\boldsymbol{v}_{2}^{2}\right)
\end{array}\right)
$$

\footnotetext{
${ }^{1}$ We concentrate here on the case with real parameters. For the case of complex parameters see Refs. [28,29] and references therein.
} 
$m_{1}, m_{2}, m_{12}$ denote the soft SUSY-breaking parameters in the Higgs sector, and $g_{2}, g_{1}$ are the $\mathrm{SU}(2)$ and $\mathrm{U}(1)$ gauge couplings, respectively. The mass eigenstates in lowest order in the charged sector follow from unitary transformations on the original fields,

$$
\left(\begin{array}{l}
H^{ \pm} \\
G^{ \pm}
\end{array}\right)=\left(\begin{array}{cc}
-\sin \beta & \cos \beta \\
\cos \beta & \sin \beta
\end{array}\right) \cdot\left(\begin{array}{c}
\phi_{1}^{ \pm} \\
\phi_{2}^{ \pm}
\end{array}\right) .
$$

This yields the (square of the) mass eigenvalue for the charged Higgs boson, $m_{H^{ \pm}}^{2}$, as given by Eq. (1). Quantum corrections substantially modify the tree-level mass. The charged Higgs boson pole mass, $M_{H^{ \pm}}$, including higherorder contributions entering via the renormalized charged Higgs boson self-energy, $\hat{\Sigma}_{H^{+} H^{-}}$, is obtained by solving the equation

$$
p^{2}-m_{H^{ \pm}}^{2}+\hat{\Sigma}_{H^{+} H^{-}}\left(p^{2}\right)=0 .
$$

This yields $M_{H^{ \pm}}^{2}$ as the real part of the complex zero of Eq. (5). The renormalized charged Higgs boson self-energy, $\hat{\Sigma}_{H^{+} H^{-}}$, is composed of the unrenormalized self-energy, $\Sigma_{H^{+} H^{-}}$, and counterterm contributions as specified below. In perturbation theory, the self-energy is expanded as follows:

$$
\Sigma\left(p^{2}\right)=\Sigma^{(1)}\left(p^{2}\right)+\Sigma^{(2)}\left(p^{2}\right)+\cdots,
$$

in terms of the $i$ th-order contributions $\Sigma^{(i)}$, and analogously for the renormalized quantities. Details for the one-loop self-energies are given below in Sec. II B, and for the two-loop contributions in Sec. II C.

A possible mixing with the charged Goldstone boson would contribute to the prediction for the charged Higgs boson mass from two-loop order onwards via terms of the form $\left(\hat{\Sigma}_{H^{ \pm} G^{\mp}}^{(1)}\left(p^{2}\right)\right)^{2}$. The mixing contributions with $G^{ \pm}$ yield a two-loop contribution that is subleading compared to the leading terms at $\mathcal{O}\left(\alpha_{t} \alpha_{s}\right)$ that we take into account, as described in Sec. IIC. Consequently, we neglect those two-loop Higgs-Goldstone mixing contributions throughout our analysis.

\section{B. One-loop corrections}

Here we review the calculation of the full one-loop corrections to $M_{H^{ \pm}}$, following Refs. [28,64]. All selfenergies and renormalization constants are understood to be one-loop quantities, dropping the order index. Renormalized self-energies, $\hat{\Sigma}\left(p^{2}\right)$, can be expressed in terms of the corresponding unrenormalized self-energies, $\Sigma\left(p^{2}\right)$, the field-renormalization constants, and the mass counterterms. For the charged Higgs boson self-energy entering Eq. (5) this expression reads

$\hat{\Sigma}_{H^{+} H^{-}}\left(p^{2}\right)=\Sigma_{H^{+} H^{-}}\left(p^{2}\right)+\delta Z_{H^{+} H^{-}}\left(p^{2}-m_{H^{ \pm}}^{2}\right)-\delta m_{H^{ \pm}}^{2}$.

The independent mass parameters are renormalized according to

$$
M_{A}^{2} \rightarrow M_{A}^{2}+\delta M_{A}^{2}, \quad M_{W}^{2} \rightarrow M_{W}^{2}+\delta M_{W}^{2},
$$

while the mass counterterm for the charged Higgs boson, arising from $m_{H^{ \pm}}^{2} \rightarrow m_{H^{ \pm}}^{2}+\delta m_{H^{ \pm}}^{2}$, is a dependent quantity. It is given in terms of the counterterms for $M_{A}$ and $M_{W}$ by

$$
\delta m_{H^{ \pm}}^{2}=\delta M_{A}^{2}+\delta M_{W}^{2} .
$$

We renormalize the $W$ boson and the $C P$-odd Higgs boson masses on shell, yielding the mass counterterms

$\delta M_{W}^{2}=\operatorname{Re} \Sigma_{W W}\left(M_{W}^{2}\right), \quad \delta M_{A}^{2}=\operatorname{Re} \Sigma_{A A}\left(M_{A}^{2}\right)$,

where $\Sigma_{W W}$ is the transverse part of the $W$ boson selfenergy.

For field renormalization, required for finite self-energies at arbitrary values of the external momentum $p^{2}$, we assign one field-renormalization constant for each Higgs doublet,

$$
\begin{gathered}
\mathcal{H}_{1} \rightarrow\left(1+\frac{1}{2} \delta Z_{\mathcal{H}_{1}}\right) \mathcal{H}_{1}, \\
\mathcal{H}_{2} \rightarrow\left(1+\frac{1}{2} \delta Z_{\mathcal{H}_{2}}\right) \mathcal{H}_{2} .
\end{gathered}
$$

For the charged Higgs field this implies

$$
H^{ \pm} \rightarrow\left(1+\frac{1}{2} \delta Z_{H^{+} H^{-}}\right) H^{ \pm}
$$

with

$$
\delta Z_{H^{+} H^{-}}=\sin ^{2} \beta \delta Z_{\mathcal{H}_{1}}+\cos ^{2} \beta \delta Z_{\mathcal{H}_{2}} .
$$

For the determination of the field-renormalization constants we adopt the $\overline{\mathrm{DR}}$ scheme,

$$
\begin{aligned}
\delta Z_{\mathcal{H}_{1}} & =\delta Z_{\mathcal{H}_{1}}^{\overline{\mathrm{DR}}}=-\left[\operatorname{Re} \Sigma_{H H \mid \alpha=0}^{\prime}\right]^{\mathrm{div}}, \\
\delta Z_{\mathcal{H}_{2}} & =\delta Z_{\mathcal{H}_{2}}^{\overline{\mathrm{DR}}}=-\left[\operatorname{Re} \Sigma_{h h \mid \alpha=0}^{\prime}\right]^{\mathrm{div}},
\end{aligned}
$$

i.e. the renormalization constants consist of divergent parts only, see the discussion in Ref. [28]. $\Sigma_{\phi \phi \mid \alpha=0}^{\prime}(\phi=h, H)$ denotes the derivative of the unrenormalized self-energies of the neutral $C P$-even Higgs bosons, with the mixing angle $\alpha$ set to zero. As default value of the renormalization scale we have chosen $\mu^{\overline{\mathrm{DR}}}=m_{t}$.

For the self-energies as specified in Eq. (7) we have evaluated the complete one-loop contributions with the help of the programs FeynArts [65] and FormCalc [66]. As regularization scheme we have used constrained differential regularization [67], which has been shown to be equivalent to dimensional reduction [68] at the one-loop level [66], thus preserving supersymmetry [69,70]. The corresponding Feynman diagrams for the charged Higgs boson (and similarly for the $W$ boson, where additional diagrams with gauge boson and ghost loops contribute) are shown in Fig. 1. The diagrams for the neutral Higgs bosons, entering $\delta M_{A}^{2}$ and $\delta Z_{\mathcal{H}_{1}}, \delta Z_{\mathcal{H}_{2}}$ (i.e. the neutral Higgs boson self-energies), are depicted in Fig. 2. 

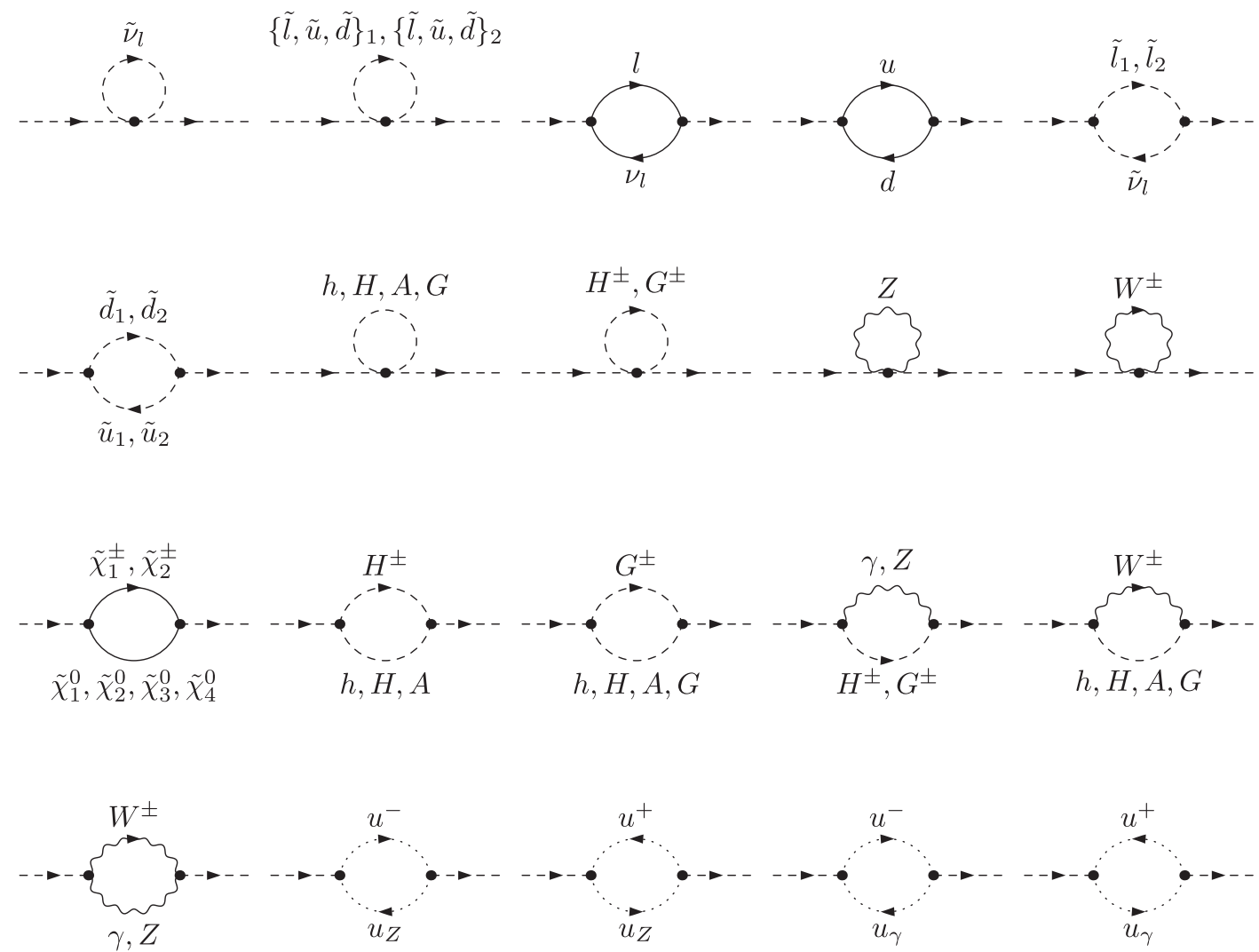

FIG. 1. Generic Feynman diagrams for the $H^{ \pm}$self-energy $(l=\{e, \mu, \tau\}, d=\{d, s, b\}, u=\{u, c, t\})$. Similar diagrams for the $W$ boson self-energy are obtained by replacing the external Higgs boson by a $W$ boson; not all combinations of particle insertions exist.

\section{Two-loop corrections}

We now turn to the $\mathcal{O}\left(\alpha_{t} \alpha_{s}\right)$ corrections at the two-loop level. Again, we drop the order index for all Higgs boson and SM gauge boson self-energies and renormalization constants, which are in this section understood to be of two-loop order. The $\mathcal{O}\left(\alpha_{t} \alpha_{s}\right)$ terms are obtained in the limit of vanishing gauge couplings and by neglecting the dependence on the external momentum [35], keeping only terms $\propto h_{t}^{2} \alpha_{s}$, with the top Yukawa coupling $h_{t}$ as defined above. We neglect the bottom Yukawa coupling in the two-loop Higgs boson self-energies. In this approximation, the counterterm for $M_{A}$ is determined as follows:

$$
\delta M_{A}^{2}=\Sigma_{A A}(0),
$$

while the renormalization constants $\delta M_{W}^{2}$ and $\delta Z_{H^{+} H^{-}}$do not contribute,

$$
\delta M_{W}^{2}=0, \quad \delta Z_{H^{+} H^{-}}=0 .
$$

Consequently, the two-loop contribution to the renormalized $H^{ \pm}$self-energy can be written in the following way:

$\hat{\Sigma}_{H^{+} H^{-}}(0)=\Sigma_{H^{+} H^{-}}(0)-\delta m_{H^{ \pm}}^{2} \quad$ with $\quad \delta m_{H^{ \pm}}^{2}=\delta M_{A}^{2}$.

From Eq. (5) we get the two-loop correction to the charged Higgs boson mass,

$$
\Delta m_{H^{ \pm}}^{2,2 \text {-loop }}=\Sigma_{A A}(0)-\Sigma_{H^{+} H^{-}}(0),
$$

with the self-energies evaluated at the two-loop level.

We thus have to evaluate the $\mathcal{O}\left(\alpha_{t} \alpha_{s}\right)$ contributions to the $H^{ \pm}$and $A$ self-energies. Examples of generic Feynman diagrams for the $H^{ \pm}$self-energy are depicted in Fig. 3, and in Fig. 4 for the $A$ boson self-energy. These contributions have been evaluated using the packages FeynArts [65] and TwoCalc [71].

\section{Subloop renormalization in the scalar top/bottom sector}

Besides the computation of the genuine two-loop diagrams at $\mathcal{O}\left(\alpha_{t} \alpha_{s}\right)$ for the self-energies, one-loop renormalization is required for the $\tilde{t}$ and $\tilde{b}$ sector providing the counterterms for one-loop subrenormalization. This yields additional diagrams with counterterm insertions; examples are the fourth diagrams in Figs. 3 and 4. The bilinear part of the $\tilde{t}$ and $\tilde{b}$ Lagrangian,

$$
\mathcal{L}_{\tilde{t} / \tilde{b} \text { mass }}=-\left(\tilde{t}_{L}^{\dagger}, \tilde{t}_{R}^{\dagger}\right) \mathbf{M}_{\tilde{t}}\left(\begin{array}{c}
\tilde{t}_{L} \\
\tilde{t}_{R}
\end{array}\right)-\left(\tilde{b}_{L}^{\dagger}, \tilde{b}_{R}^{\dagger}\right) \mathbf{M}_{\tilde{b}}\left(\begin{array}{c}
\tilde{b}_{L} \\
\tilde{b}_{R}
\end{array}\right),
$$

contains the stop and sbottom mass matrices $\mathbf{M}_{\tilde{t}}$ and $\mathbf{M}_{\tilde{b}}$, given by 

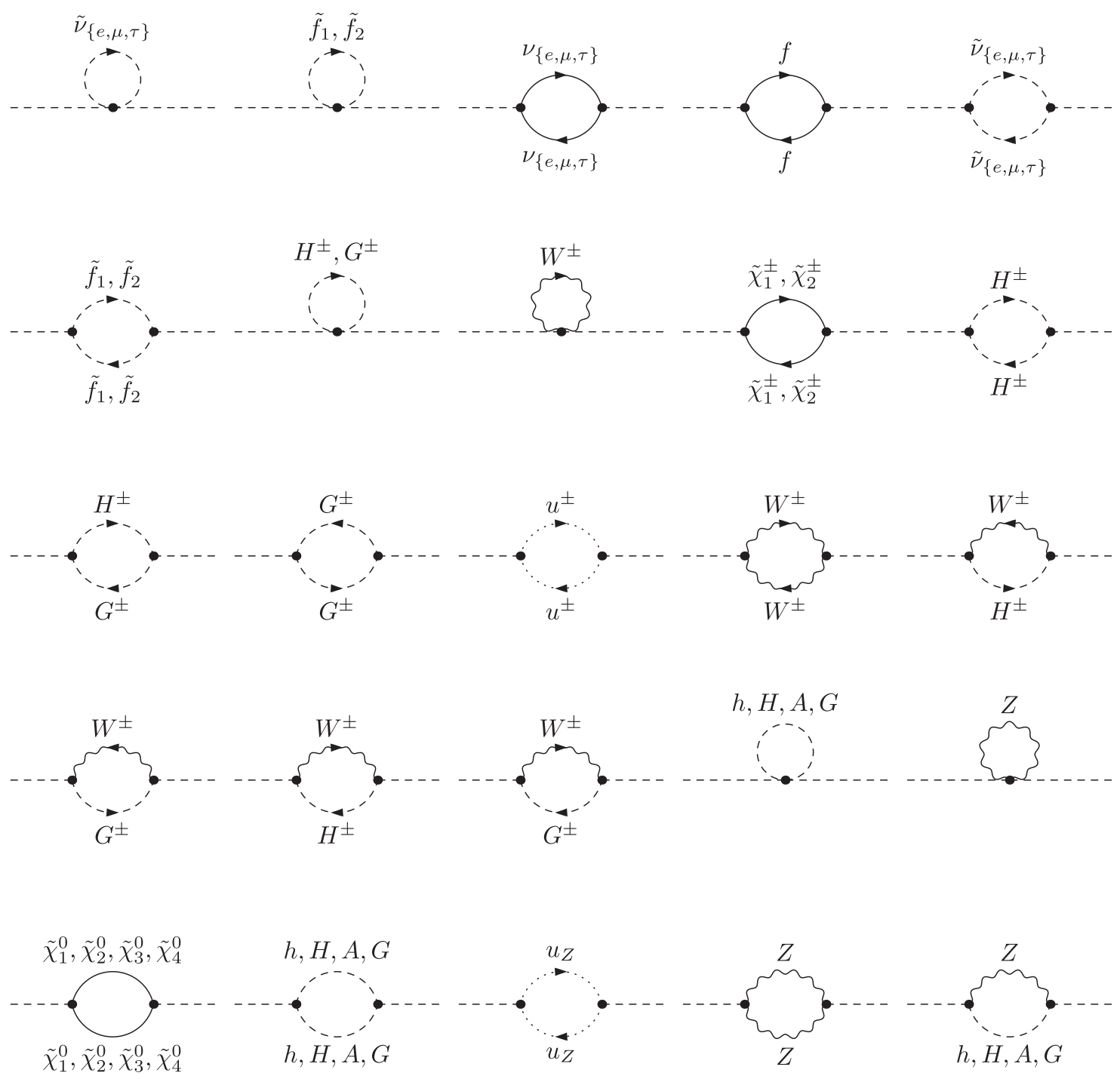

FIG. 2. Generic Feynman diagrams for the $h, H, A$, self-energies $(f=\{e, \mu, \tau, d, s, b, u, c, t\})$. Not all combinations of particle insertions exist for all neutral Higgs bosons.

$$
\mathbf{M}_{\tilde{q}}=\left(\begin{array}{cc}
M_{L}^{2}+m_{q}^{2} & m_{q} X_{q} \\
m_{q} X_{q} & M_{\tilde{q}_{R}}^{2}+m_{q}^{2}
\end{array}\right),
$$

with

$$
X_{q}=A_{q}-\mu \kappa, \quad \kappa=\{\cot \beta, \tan \beta\} \quad \text { for } q=\{t, b\} .
$$

Here $M_{L}^{2}, M_{\tilde{q}_{R}}^{2}$ are soft-breaking parameters, where $M_{L}^{2}$ is the same for $\mathbf{M}_{\tilde{t}}$ and $\mathbf{M}_{\tilde{b}}$ (see below), and $A_{q}$ is the trilinear soft-breaking parameter. The D-terms do not contribute to $\mathcal{O}\left(\alpha_{t} \alpha_{s}\right)$ and therefore have to be neglected in the calculation of the stop mass values entering the contribution of this order [29]. The mass matrix can be diagonalized with the help of a unitary transformation $\mathbf{U}_{\tilde{q}}$, which can be parametrized by a mixing angle $\theta_{\tilde{q}}$,

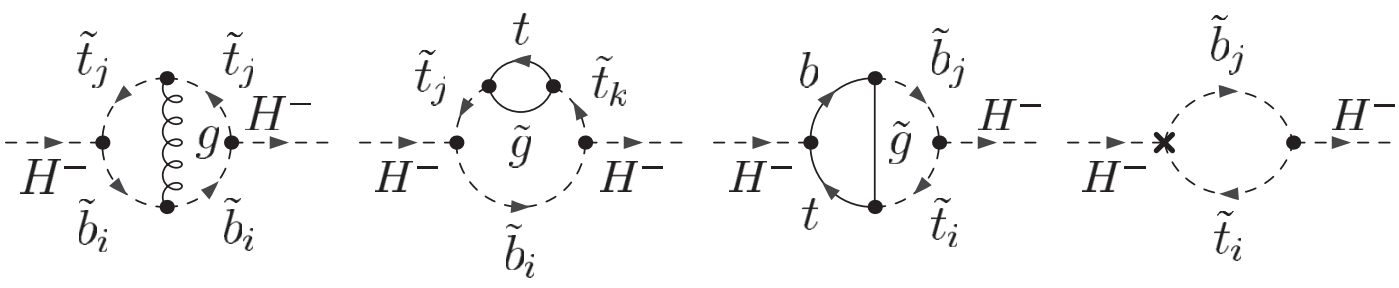

FIG. 3. Examples of generic two-loop diagrams and diagrams with counterterm insertion for the charged Higgs boson self-energy $(i, j, k=1,2)$. 


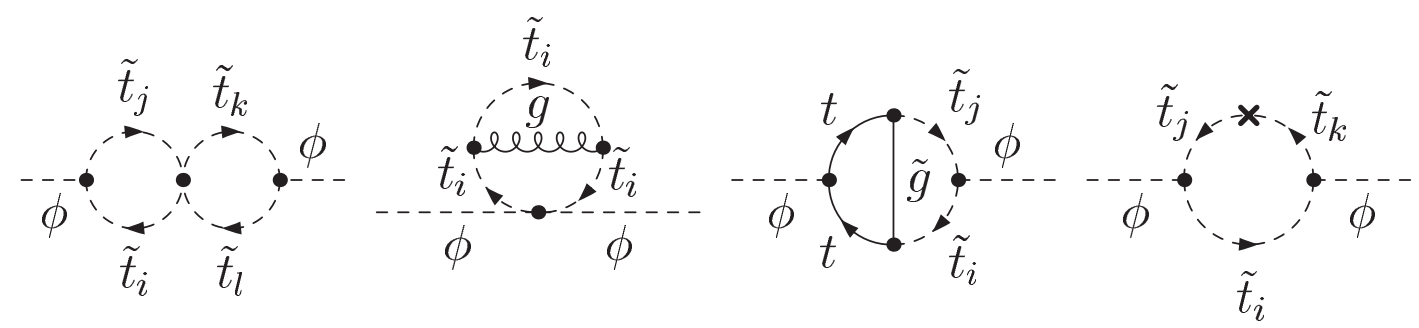

FIG. 4. Examples of generic two-loop diagrams and diagrams with counterterm insertion for the $A$ boson self-energy $(i, j, k, l=1,2)$.

$$
\begin{aligned}
\mathbf{D}_{\tilde{q}} & =\mathbf{U}_{\tilde{q}} \mathbf{M}_{\tilde{q}} \mathbf{U}_{\tilde{q}}^{\dagger}=\left(\begin{array}{cc}
m_{\tilde{q}_{1}}^{2} & 0 \\
0 & m_{\tilde{q}_{2}}^{2}
\end{array}\right), \\
\mathbf{U}_{\tilde{q}} & =\left(\begin{array}{ll}
U_{\tilde{q}_{11}} & U_{\tilde{q}_{12}} \\
U_{\tilde{q}_{21}} & U_{\tilde{q}_{22}}
\end{array}\right)=\left(\begin{array}{cc}
\cos \theta_{\tilde{q}} & \sin \theta_{\tilde{q}} \\
-\sin \theta_{\tilde{q}} & \cos \theta_{\tilde{q}}
\end{array}\right) .
\end{aligned}
$$

We follow here the renormalization prescription used in Refs. [72,73]. In the MSSM the $t / \tilde{t}$ sector is described in terms of four real parameters (where we assume that $\mu$ and $\tan \beta$ are defined via other sectors): the real soft SUSY-breaking parameters $M_{L}^{2}$ and $M_{\tilde{t}_{R}}^{2}$, the trilinear coupling $A_{t}$, and the top Yukawa coupling $h_{t}$. Instead of the quantities $M_{L}^{2}, M_{\tilde{t}_{R}}^{2}$ and $h_{t}$, in the on-shell scheme applied in this paper we choose the on-shell squark masses $m_{\tilde{t}_{1}}^{2}, m_{\tilde{t}_{2}}^{2}$ and the top-quark mass $m_{t}$ as independent parameters. It should furthermore be noted that the counterterms are evaluated at $\mathcal{O}\left(\alpha_{s}\right)$, such as to yield the desired $\mathcal{O}\left(\alpha_{t} \alpha_{s}\right)$ contributions when combined with the one-loop diagrams with counterterm insertion.

The following renormalization conditions are imposed:

(i) The top-quark mass is defined on shell, yielding the mass counterterm $\delta m_{t}$,

$$
\begin{aligned}
\delta m_{t}= & \frac{1}{2} m_{t}\left(\operatorname{Re} \Sigma_{t}^{L}\left(m_{t}^{2}\right)+\operatorname{Re} \Sigma_{t}^{R}\left(m_{t}^{2}\right)\right. \\
& \left.+2 \operatorname{Re} \Sigma_{t}^{S}\left(m_{t}^{2}\right)\right)
\end{aligned}
$$

referring to the Lorentz decomposition of the selfenergy $\Sigma_{t}$

$$
\Sigma_{t}(k)=\not k \omega_{-} \Sigma_{t}^{L}\left(k^{2}\right)+\not k \omega_{+} \Sigma_{t}^{R}\left(k^{2}\right)+m_{t} \Sigma_{t}^{S}\left(k^{2}\right)
$$

into a left-handed, a right-handed, and a scalar part, $\Sigma_{t}^{L}, \Sigma_{t}^{R}, \Sigma_{t}^{S}$, respectively.

(ii) The stop masses are also determined via on-shell conditions [35,72], yielding

$$
\delta m_{\tilde{t}_{i}}^{2}=\operatorname{Re} \Sigma_{\tilde{t}_{i i}}\left(m_{\tilde{t}_{i}}^{2}\right) \quad \text { with } \quad i=1,2
$$

(iii) The third condition affects the trilinear coupling $A_{t}$. Rewriting the squark mass matrix in terms of the mass eigenvalues and the mixing angle using Eq. (22),

$$
\mathbf{M}_{\tilde{t}}=\left(\begin{array}{cc}
\cos ^{2} \theta_{\tilde{t}} m_{\tilde{t}_{1}}^{2}+\sin ^{2} \theta_{\tilde{t}} m_{\tilde{t}_{2}}^{2} & \sin \theta_{\tilde{t}} \cos \theta_{\tilde{t}}\left(m_{\tilde{t}_{1}}^{2}-m_{\tilde{t}_{2}}^{2}\right) \\
\sin \theta_{\tilde{t}} \cos \theta_{\tilde{t}}\left(m_{\tilde{t}_{1}}^{2}-m_{\tilde{t}_{2}}^{2}\right) & \sin ^{2} \theta_{\tilde{t}} m_{\tilde{t}_{1}}^{2}+\cos ^{2} \theta_{\tilde{t}} m_{\tilde{t}_{2}}^{2}
\end{array}\right),
$$

yields the counterterm matrix $\delta \mathbf{M}_{\tilde{t}}$ by introducing counterterms $\delta m_{\tilde{t}_{1}}^{2}, \delta m_{\tilde{t}_{2}}^{2}$ for the masses and $\delta \theta_{\tilde{t}}$ for the angle. One obtains the counterterm for the offdiagonal contribution in the stop sector,

$$
\left(m_{\tilde{t}_{1}}^{2}-m_{\tilde{t}_{2}}^{2}\right) \delta \theta_{\tilde{t}}=\left[\mathbf{U}_{\tilde{t}} \delta \mathbf{M}_{\tilde{t}} \mathbf{U}_{\tilde{t}}^{\dagger}\right]_{12} \equiv \delta Y_{\tilde{t}}
$$

for which the following renormalization condition has been used [72,73]:

$$
\delta Y_{\tilde{t}}=\frac{1}{2}\left[\operatorname{Re} \Sigma_{\tilde{t}_{12}}\left(m_{\tilde{t}_{1}}^{2}\right)+\operatorname{Re} \Sigma_{\tilde{t}_{12}}\left(m_{\tilde{t}_{2}}^{2}\right)\right] .
$$

Finally we derive the relation between the counterterms $\delta A_{t}$ and $\delta \theta_{\tilde{t}}$. The two counterterms are mutually related via Eq. (20) and (26). The off-diagonal entries of the corresponding counterterm matrices yield

$$
\begin{aligned}
\left(A_{t}-\mu \cot \beta\right) \delta m_{t}+m_{t} \delta A_{t}= & \sin \theta_{\tilde{t}} \cos \theta_{\tilde{t}}\left(\delta m_{\tilde{t}_{1}}^{2}-\delta m_{\tilde{t}_{2}}^{2}\right) \\
& +\left(\cos ^{2} \theta_{\tilde{t}}-\sin ^{2} \theta_{\tilde{t}}\right) \delta Y_{t} .
\end{aligned}
$$

As a result, we obtain for $\delta A_{t}$

$$
\begin{aligned}
\delta A_{t}= & \frac{1}{m_{t}}\left[\frac{1}{2} \sin 2 \theta_{\tilde{t}}\left(\delta m_{\tilde{t}_{1}}^{2}-\delta m_{\tilde{t}_{2}}^{2}\right)+\cos 2 \theta_{\tilde{t}} \delta Y_{t}\right. \\
& \left.-\frac{1}{2 m_{t}} \sin 2 \theta_{\tilde{t}}\left(m_{\tilde{t}_{1}}^{2}-m_{\tilde{t}_{2}}^{2}\right) \delta m_{t}\right] .
\end{aligned}
$$

In the $b / \tilde{b}$ sector, we also encounter four real parameters (with $\mu$ and $\tan \beta$ defined via other sectors): the soft-breaking mass parameters $M_{L}^{2}$ and $M_{\tilde{b}_{R}}^{2}$, the trilinear coupling $A_{b}$, and the bottom Yukawa coupling $h_{b}$ or the 
$b$-quark mass, respectively (which is neglected for the set of two-loop corrections presented in this paper). SU(2) invariance requires the "left-handed" soft-breaking parameters in the stop and the sbottom sector to be identical (denoted as $M_{L}^{2}$ ). With the approximations described above this yields, e.g., $m_{\tilde{b}_{L}}=M_{L}$. In the evaluation of the $\mathcal{O}\left(\alpha_{t} \alpha_{s}\right)$ contributions to the Higgs boson self-energies, the counterterms of the sbottom sector appear only in the self-energy of the charged Higgs boson. In our approximation for the two-loop contributions, where the $b$-quark mass is neglected, $\tilde{b}_{L}$ and $\tilde{b}_{R}$ do not mix, and $\tilde{b}_{R}$ decouples and does not contribute. The two-loop contribution to the charged Higgs boson self-energy thus depends only on a single parameter of the sbottom sector, which can be chosen as the squark mass $m_{\tilde{b}_{L}}$. By means of $\mathrm{SU}(2)$ invariance, the corresponding mass counterterm is already determined:

$\delta m_{\tilde{b}_{L}}^{2}=\cos ^{2} \theta_{\tilde{t}} \delta m_{\tilde{t}_{1}}^{2}+\sin ^{2} \theta_{\tilde{t}} \delta m_{\tilde{t}_{2}}^{2}-\sin 2 \theta_{\tilde{t}} \delta Y_{t}-2 m_{t} \delta m_{t}$.

With the set of renormalization constants determined in Eqs. (23), (25), (30), and (31) the counterterms arising from the one-loop subrenormalization of the stop and sbottom sectors are fully specified.

Finally, at $\mathcal{O}\left(\alpha_{t} \alpha_{s}\right)$ gluinos appear as virtual particles at the two-loop level; hence, no renormalization in the gluino sector is needed. The corresponding soft-breaking gluino mass parameter is denoted $M_{3}$. In the case of real MSSM parameters considered here the gluino mass is given as $m_{\tilde{g}}=M_{3}$.

\section{E. Higher-order corrections in the $\boldsymbol{b} / \tilde{b}$ sector}

We furthermore include in our prediction for $M_{H^{ \pm}}$corrections beyond the one-loop level originating from the bottom/sbottom sector contributions to $\Sigma_{A A}$ and $\Sigma_{H^{+} H^{-}}$. Potentially large higher-order effects proportional to $\tan \beta$ can arise in the relation between the bottom-quark mass and the bottom Yukawa coupling as described in Refs. [50,51]. The leading $\tan \beta$-enhanced contribution in the limit of heavy SUSY masses can be expressed in terms of a quantity $\Delta_{b}$ and resummed to all orders using an effective Lagrangian approach. The relevant part of the effective Lagrangian is given by

$$
\begin{aligned}
\mathcal{L}= & \frac{g}{2 M_{W}} \frac{\bar{m}_{b}}{1+\Delta_{b}}\left[\tan \beta A i \bar{b} \gamma_{5} b\right. \\
& \left.+\sqrt{2} V_{t b} \tan \beta H^{+} \bar{t}_{L} b_{R}\right]+ \text { H.c. }
\end{aligned}
$$

Here

$$
\begin{gathered}
\bar{m}_{b}^{\overline{\mathrm{DR}}, \mathrm{SM}}(Q)=\bar{m}_{b}^{\overline{\mathrm{MS}}, \mathrm{SM}}(Q)\left(1-\frac{\alpha_{s}}{3 \pi}\right), \\
\bar{m}_{b}=\bar{m}_{b}^{\overline{\mathrm{DR}}, \mathrm{SM}}\left(Q=m_{t}\right)\left(1+\frac{1}{2}\left(\Sigma_{b, \text { fin }}^{L}\left(m_{b}\right)+\sum_{b, \text { fin }}^{R}\left(m_{b}\right)\right)\right) .
\end{gathered}
$$

$\bar{m}_{b}^{\overline{\mathrm{DR}}, \mathrm{SM}}(Q)$ denotes a running bottom-quark mass at the scale $Q$ in the $\overline{\mathrm{DR}}$ scheme that incorporates SM QCD corrections (i.e., no SUSY QCD effects are included in the running). The corresponding mass in the $\overline{\mathrm{MS}}$ scheme is denoted by $\bar{m}_{b}^{\overline{\mathrm{MS}}, \mathrm{SM}}(Q) . \Sigma_{b, \text { fin }}^{L}\left(m_{b}\right)$ and $\Sigma_{b, \text { fin }}^{R}\left(m_{b}\right)$ are the finite parts of the self-energies defined in analogy to Eq. (24). $V_{t b}$ denotes the $(3,3)$ element of the CabibboKobayashi-Maskawa (CKM) matrix. In the numerical evaluations performed with the program FeynHiggs below we use $\bar{m}_{b}^{\overline{\mathrm{DR}}, \mathrm{SM}}\left(Q=m_{t}\right) \approx 2.68 \mathrm{GeV}$.

The leading $\tan \beta$-enhanced one-loop contribution in the limit of heavy SUSY masses takes the simple form [49]

$$
\begin{aligned}
\Delta_{b}= & \frac{2 \alpha_{s}}{3 \pi} m_{\tilde{g}} \mu \tan \beta \times I\left(m_{\tilde{b}_{1}}, m_{\tilde{b}_{2}}, m_{\tilde{g}}\right) \\
& +\frac{\alpha_{t}}{4 \pi} A_{t} \mu \tan \beta \times I\left(m_{\tilde{t}_{1}}, m_{\tilde{t}_{2}}, \mu\right)+\cdots,
\end{aligned}
$$

where $\alpha_{s}$ is evaluated at the scale $\sqrt{m_{\tilde{b}_{1}} m_{\tilde{b}_{2}}}$, and the function $I$ is given by

$$
\begin{aligned}
I(a, b, c)= & \frac{1}{\left(a^{2}-b^{2}\right)\left(b^{2}-c^{2}\right)\left(a^{2}-c^{2}\right)} \\
& \times\left(a^{2} b^{2} \log \frac{a^{2}}{b^{2}}+b^{2} c^{2} \log \frac{b^{2}}{c^{2}}+c^{2} a^{2} \log \frac{c^{2}}{a^{2}}\right) \\
\sim & \frac{1}{\max \left(a^{2}, b^{2}, c^{2}\right)} .
\end{aligned}
$$

The ellipses in Eq. (35) denote subleading terms that we take over from Ref. [74]. Expanded up to one-loop order, the effective mass $\bar{m}_{b} /\left(1+\Delta_{b}\right)$ is close to the $\overline{\mathrm{DR}}$ mass (including SUSY contributions in the running), see Refs. [45,73]. A recent two-loop calculation of $\Delta_{b}$ can be found in Ref. [75].

\section{APPROXIMATION FOR THE TWO-LOOP CORRECTIONS}

In Sec. II we have described the approximations for getting the two-loop $\mathcal{O}\left(\alpha_{t} \alpha_{s}\right)$ terms, which can be written as terms proportional to $m_{t}^{4}$. It is well known that for the neutral Higgs bosons this procedure indeed yields the dominant part of the one-loop [30-33] and the two-loop corrections $[34,35]$.

For the charged Higgs boson mass, $M_{H^{ \pm}}$, the described procedure provides the analogous contribution to the mass shift as well,

$$
\Delta M_{H^{ \pm}}^{2} \sim \frac{m_{t}^{4}}{v^{2}} \sim \frac{m_{t}^{4}}{M_{W}^{2}} .
$$

There are, however, other contributions of similar structure at the one-loop level [59-63],

$$
\begin{gathered}
\Delta M_{H^{ \pm}}^{2} \sim \frac{m_{t}^{2} m_{b}^{2}}{M_{W}^{2}} \quad \text { or } \quad \Delta M_{H^{ \pm}}^{2} \sim \frac{m_{t}^{4}}{M_{W}^{2}} \frac{M_{W}^{2}}{M_{\mathrm{SUSY}}^{2}} \text { or } \\
\Delta M_{H^{ \pm}}^{2} \sim \frac{m_{t}^{4}}{M_{W}^{2}} \frac{M_{A}^{2}}{M_{\mathrm{SUSY}}^{2}},
\end{gathered}
$$


which are not covered by our approximations for the two-loop terms because they would correspond to $m_{b} \neq 0$ (first), nonvanishing gauge couplings (second), and $p^{2} \neq 0$ in the $A$ self-energy (third term). This is justified for large scalar-quark mass scales $M_{\text {SUSY }}$ where the second and third type of terms are suppressed. On the other hand, the term (37) extracted by our approximation can in general be large also for large $M_{\mathrm{SUSY}}$, both at the one-loop and the two-loop level, as we will explain below.

\section{A. The one-loop case}

Applying the approximations outlined in Sec. II C at the one-loop level yields the counterterms (all quantities in this section are understood to be one-loop quantities),

$\delta M_{W}^{2}=0, \quad \delta M_{A}^{2}=\Sigma_{A A}(0), \quad \delta Z_{H^{+} H^{-}}=0$,

and thus
$\hat{\Sigma}_{H^{+} H^{-}}=\Sigma_{H^{+} H^{-}}(0)-\delta m_{H^{ \pm}}^{2} \quad$ with $\quad \delta m_{H^{ \pm}}^{2}=\delta M_{A}^{2}$.

From Eq. (5) we get the one-loop corrected value of the charged Higgs boson mass,

$$
M_{H^{ \pm}}^{2}=m_{H^{ \pm}}^{2}+\Delta m_{H^{ \pm}}^{2},
$$

with

$$
\Delta m_{H^{ \pm}}^{2}=\Sigma_{A A}(0)-\Sigma_{H^{+} H^{-}}(0) .
$$

In the following we use the factor $c$ to simplify the notation $\left(v^{2}=v_{1}^{2}+v_{2}^{2}\right)$ :

$$
c=-\frac{3 m_{t}^{2}}{16 \pi^{2} v^{2} \tan ^{2} \beta}=-\frac{3 e^{2} m_{t}^{2}}{32 \pi^{2} s_{\mathrm{w}}^{2} M_{W}^{2} \tan ^{2} \beta} .
$$

From the third (s)quark generation, with $m_{b}=0$, one obtains the explicit expressions

$$
\begin{aligned}
\Sigma_{A A}(0)= & c\left\{2 A_{0}\left(m_{t}\right)-A_{0}\left(m_{\tilde{t}_{2}}\right)-A_{0}\left(m_{\tilde{t}_{1}}\right)-\left(A_{t}+\mu \tan \beta\right)^{2} \frac{A_{0}\left(m_{\tilde{t}_{2}}\right)-A_{0}\left(m_{\tilde{t}_{1}}\right)}{m_{\tilde{t}_{2}}^{2}-m_{\tilde{t}_{1}}^{2}}\right\}, \\
\Sigma_{H^{+} H^{-}}(0)= & c\left\{2 A_{0}\left(m_{t}\right)-A_{0}\left(m_{\tilde{b}_{1}}\right)-s_{\tilde{t}}^{2} A_{0}\left(m_{\tilde{t}_{1}}\right)-c_{\tilde{t}}^{2} A_{0}\left(m_{\tilde{t}_{2}}\right)-\left(c_{\tilde{t}} m_{t}+s_{\tilde{t}}\left(A_{t}+\mu \tan \beta\right)\right)^{2} \frac{A_{0}\left(m_{\tilde{t}_{1}}\right)-A_{0}\left(m_{\tilde{b}_{1}}\right)}{m_{\tilde{t}_{1}}^{2}-m_{\tilde{b}_{1}}^{2}}\right. \\
& \left.-\left(s_{\tilde{t}} m_{t}-c_{\tilde{t}}\left(A_{t}+\mu \tan \beta\right)\right)^{2} \frac{A_{0}\left(m_{\tilde{t}_{2}}\right)-A_{0}\left(m_{\tilde{b}_{1}}\right)}{m_{\tilde{t}_{2}}^{2}-m_{\tilde{b}_{1}}^{2}}\right\} .
\end{aligned}
$$

Here we use the abbreviation $s_{\tilde{t}} \equiv \sin \theta_{\tilde{t}}, c_{\tilde{t}} \equiv \cos \theta_{\tilde{t}}$, and the one-loop integral function $A_{0}(m)$ is defined as in Ref. [76]. In the approximation of $m_{b}=0$ and neglected gauge couplings the mass of the left-handed sbottom is given by

$$
m_{\tilde{b}_{L}}^{2}=c_{\tilde{t}}^{2} m_{\tilde{t}_{1}}^{2}+s_{\tilde{t}}^{2} m_{\tilde{t}_{2}}^{2}-m_{t}^{2}\left(=M_{L}^{2}\right) .
$$

Using these relations results then in the following expression for $\Delta m_{H^{ \pm}}^{2}$ :

$$
\begin{aligned}
\Delta m_{H^{ \pm}}^{2}= & -c\left\{m_{\tilde{b}_{L}}^{2}\left[1+\frac{\left[\left(A_{t}+\mu \tan \beta\right) s_{\tilde{t}}+m_{t} c_{\tilde{t}}\right]^{2}}{m_{\tilde{b}_{L}}^{2}-m_{\tilde{t}_{1}}^{2}}+\frac{\left[m_{t} s_{\tilde{t}}-c_{\tilde{t}}\left(A_{t}+\mu \tan \beta\right)\right]^{2}}{m_{\tilde{b}_{L}}^{2}-m_{\tilde{t}_{2}}^{2}}\right] \log \left(\frac{m_{\tilde{b}_{L}}^{2}}{m_{\tilde{t}_{1}}^{2}}\right)\right. \\
& \left.+m_{\tilde{t}_{2}}^{2}\left[-s_{\tilde{t}}^{2}+\frac{\left(A_{t}+\mu \tan \beta\right)^{2}}{m_{\tilde{t}_{1}}^{2}-m_{\tilde{t}_{2}}^{2}}-\frac{\left[m_{t} s_{\tilde{t}}-c_{\tilde{t}}\left(A_{t}+\mu \tan \beta\right)\right]^{2}}{m_{\tilde{b}_{L}}^{2}-m_{\tilde{t}_{2}}^{2}}\right] \log \left(\frac{m_{\tilde{t}_{2}}^{2}}{m_{\tilde{t}_{1}}^{2}}\right)\right\} .
\end{aligned}
$$

It is possible to eliminate the dependence on $A_{t}$ and $\theta_{\tilde{t}}$ from the expression of the charged Higgs boson mass correction,

$$
\begin{aligned}
\Delta m_{H^{ \pm}}^{2}= & \frac{c m_{t}^{2}}{\left(m_{\tilde{b}_{L}}^{2}-m_{\tilde{t}_{1}}^{2}\right)\left(m_{\tilde{b}_{L}}^{2}-m_{\tilde{t}_{2}}^{2}\right)} \frac{\mu^{2}}{\sin ^{2} \beta \cos ^{2} \beta} \\
& \times\left[m_{\tilde{b}_{L}}^{2} \log \left(\frac{m_{\tilde{b}_{L}}^{2}}{m_{\tilde{t}_{1}} m_{\tilde{t}_{2}}}\right)-\frac{m_{\tilde{t}_{1}}^{2}\left(m_{\tilde{b}_{L}}^{2}-m_{\tilde{t}_{2}}^{2}\right)+m_{\tilde{t}_{2}}^{2}\left(m_{\tilde{b}_{L}}^{2}-m_{\tilde{t}_{1}}^{2}\right)}{m_{\tilde{t}_{1}}^{2}-m_{\tilde{t}_{2}}^{2}} \log \left(\frac{m_{\tilde{t}_{1}}}{m_{\tilde{t}_{2}}}\right)\right] .
\end{aligned}
$$

This shows explicitly the $m_{t}^{4}$ dependence of this contribution as well as the overall factor $\mu^{2} / \cos ^{2} \beta$, which strongly determines the phenomenology of the $\mathcal{O}\left(\alpha_{t}\right)$ charged Higgs boson mass corrections. In the following, we specify the analytic result, assuming a common SUSY mass scale $M_{L}=M_{\tilde{t}_{R}}=: M_{\text {SUSY }}$. With this simplification one obtains

$$
\begin{aligned}
& m_{\tilde{t}_{1}}^{2}=M_{\mathrm{SUSY}}^{2}+m_{t}^{2}-m_{t}\left|X_{t}\right|, \\
& m_{\tilde{t}_{2}}^{2}=M_{\mathrm{SUSY}}^{2}+m_{t}^{2}+m_{t}\left|X_{t}\right|, \\
& m_{\tilde{b}_{L}}^{2}=M_{\mathrm{SUSY}}^{2},
\end{aligned}
$$

(and $s_{\tilde{t}}^{2}=c_{\tilde{t}}^{2}=1 / 2$ in this case). This yields 


$$
\begin{aligned}
\Delta m_{H^{ \pm}}^{2}= & \frac{c m_{t}^{2} \mu^{2}}{\sin ^{2} \beta \cos ^{2} \beta} \times \frac{1}{2 m_{t}^{2}\left|X_{t}\right|\left(X_{t}^{2}-m_{t}^{2}\right)} \times\left[m_{t}\left(M_{\mathrm{SUSY}}^{2}+m_{t}^{2}-X_{t}^{2}\right) \log \left(\frac{M_{\mathrm{SUSY}}^{2}+m_{t}\left(m_{t}-\left|X_{t}\right|\right)}{M_{\mathrm{SUSY}}^{2}+m_{t}\left(m_{t}+\left|X_{t}\right|\right)}\right)\right. \\
& \left.-M_{\mathrm{SUSY}}^{2}\left|X_{t}\right| \log \left(\frac{M_{\mathrm{SUSY}}^{4}}{M_{\mathrm{SUSY}}^{4}+2 M_{\mathrm{SUSY}}^{2} m_{t}^{2}+m_{t}^{4}-m_{t}^{2} X_{t}^{2}}\right)\right] .
\end{aligned}
$$

Expanding in inverse powers of $M_{\text {SUSY }}$ and inserting the prefactor $c$ from Eq. (43) we find

$$
\begin{aligned}
\Delta m_{H^{ \pm}}^{2} \approx & -\frac{3 e^{2} \mu^{2}}{64 \pi^{2} s_{\mathrm{w}}^{2} \sin ^{4} \beta} \frac{m_{t}^{4}}{M_{W}^{2}}\left[\frac{1}{M_{\mathrm{SUSY}}^{2}}-\frac{2 m_{t}^{2}}{3 M_{\mathrm{SUSY}}^{4}}\right. \\
& \left.+\frac{m_{t}^{2}\left(3 m_{t}^{2}+X_{t}^{2}\right)}{6 M_{\mathrm{SUSY}}^{6}}\right] .
\end{aligned}
$$

Thus one obtains the term proportional to $m_{t}^{4} / M_{W}^{2}$. In the special case of $X_{t}=0$ and restricting to the leading term in the expansion in inverse powers of $M_{\text {SUSY }}$ (vanishing stop mixing) this reduces to

$$
\Delta m_{H^{ \pm}}^{2} \approx-\frac{3 e^{2} \mu^{2}}{64 \pi^{2} s_{\mathrm{w}}^{2} \sin ^{4} \beta} \frac{m_{t}^{4}}{M_{W}^{2} m_{\tilde{t}}^{2}},
$$

where $m_{\tilde{t}}^{2} \equiv M_{\text {SUSY }}^{2}+m_{t}^{2}$. If $|\mu| \approx m_{\tilde{t}}$ this term is not suppressed by large SUSY mass scales.

\section{B. The two-loop case}

The derivation of Eqs. (50) and (51) shows that besides the $m_{t}^{2}$ in the prefactor arising from the Yukawa couplings, the second factor $\sim m_{t}^{2}$ stems from the stop mass matrix. In other words, it is induced by the $\mathrm{SU}(2)$ breaking in the MSSM quark and squark sector. Thus, the derived term $\sim m_{t}^{4} / M_{W}^{2}$ is related to the mass difference between top and bottom squarks resulting from $m_{t} / m_{b} \gg 1$. The diagrams playing the leading role here are the second and sixth Feynman diagram in Figs. 1 and 2.

Equations (50) and (51) indicate which parameter combinations of $A_{t}, \mu$ and $\tan \beta$ can give rise to a sizable $\mathcal{O}\left(\alpha_{t}\right)$ contribution to $M_{H^{ \pm}}^{2}$ and possibly constitute a large part of the full one-loop corrections. For the corresponding parameter ranges it can be expected that also the new two-loop corrections of $\mathcal{O}\left(\alpha_{t} \alpha_{s}\right)$ are sizable and should be taken into account.

At the two-loop level the $\sim m_{t}^{4}$ contributions are augmented by the corresponding term with a renormalized $m_{t}$ parameter, leading to $\sim 4 m_{t}^{3} \delta m_{t}$. The source of these corrections is still related to the $\mathrm{SU}(2)$ breaking inducing the mass difference for scalar tops and bottoms, which enters the two-loop level Higgs boson self-energies through mass-counterterm insertions, as illustrated in the fourth diagram in Fig. 4. The inserted one-loop counterterms are given by Eq. (25) for top squarks and by Eq. (31) for bottom squarks. They differ essentially by a term $2 m_{t} \delta m_{t}$, which induces an effective mass splitting between the scalar top and bottom sector at the counterterm level. The full contribution $\sim \delta m_{t}$ can be obtained by renormalizing $m_{t}$ in Eqs. (50) and (51), or by an explicit extraction of this term. In the case of vanishing stop mixing, corresponding to Eq. (51), we have checked that both calculations indeed agree. In this case they yield [keeping in mind the prefactor $c \propto m_{t}^{2}$ in Eq. (43)]

$$
\begin{aligned}
\Delta m_{H^{ \pm}}^{2,2-l o o p}, \delta \mathrm{m}_{\mathrm{t}} & \sim \frac{\left(A_{t}+\mu \tan \beta\right)^{2}}{m_{t}^{2}}\left[\delta m_{\tilde{t}}^{2} \frac{m_{t}^{2}}{m_{\tilde{t}}^{2}}-\left(\delta m_{\tilde{t}}^{2}-2 m_{t} \delta m_{t}\right) \log \left(\frac{m_{\tilde{t}}^{2}}{m_{\tilde{t}}^{2}-m_{t}^{2}}\right)\right] \\
& =\frac{\mu^{2}}{\sin ^{2} \beta \cos ^{2} \beta}\left[\frac{\delta m_{\tilde{t}}^{2}}{m_{\tilde{t}}^{2}}-\frac{\delta m_{\tilde{t}}^{2}-2 m_{t} \delta m_{t}}{m_{t}^{2}} \log \left(\frac{m_{\tilde{t}}^{2}}{m_{\tilde{t}}^{2}-m_{t}^{2}}\right)\right] \approx \frac{\mu^{2}}{2 \sin ^{2} \beta \cos ^{2} \beta}\left[\frac{4 m_{t} \delta m_{t}}{m_{\tilde{t}}^{2}}\right] .
\end{aligned}
$$

For the case of nonvanishing stop mixing, see Eq. (50), we find accordingly

$$
\begin{aligned}
\Delta m_{H^{ \pm}}^{2,2-l o o p, \delta m_{\mathrm{t}}} \sim & \frac{\mu^{2}}{\sin ^{2} \beta \cos ^{2} \beta}\left[\frac{2 m_{t}}{M_{\text {SUSY }}^{2}}-\frac{2 m_{t}^{3}}{M_{\text {SUSY }}^{4}}\right. \\
& \left.+\frac{m_{t}^{3}\left(4 m_{t}^{2}+X_{t}^{2}\right)}{2 M_{\text {SUSY }}^{6}}\right] \times \delta m_{t} .
\end{aligned}
$$

In conclusion, although the two-loop corrections to $M_{H^{ \pm}}^{2}$ covered by our approach are only part of the complete two-loop Yukawa corrections, they constitute a finite well-defined subset that can induce non-negligible mass shifts for the $H^{ \pm}$boson. Numerical examples will be given in Sec. IV B.

\section{NUMERICAL ANALYSIS}

Our results obtained in this paper extend the known results in the literature in various ways. While the one-loop result in Ref. [63] was complete, the numerical evaluation focused on particular parameter values, mostly excluded nowadays by the LEP Higgs searches [10,11,23,24]. Reference [77] focused on the mass splitting $M_{H^{ \pm}}-M_{A}$ induced by $\Delta_{b}$ effects. We perform a more general numerical analysis, including the full one-loop corrections. Furthermore for the first time explicit two-loop corrections to $M_{H^{ \pm}}$are analyzed. The higher-order corrected Higgs boson sector has been evaluated with the help of the Fortran code FeynHiggs [28,35,52,55] (current version: 2.9.4). 
The goal for the precision in predicting $M_{H^{ \pm}}$in the MSSM should be the prospective experimental resolution or better. For the LHC no dedicated study has been performed recently. Older evaluations indicate that a precision $\$ 5 \%$ might be possible in the region of large $\tan \beta$ [78]. Other studies, focusing on the $\tau \nu_{\tau}$ decay mode yielded a precision at the 1\%-2\% level [79]. At the LC for $M_{H^{ \pm}}<m_{t}$ a precision of $\sim 1 \mathrm{GeV}$ could be possible [80], while for $M_{H^{ \pm}}>m_{t}$ (but $M_{H^{ \pm}}<\sqrt{s} / 2$ ) a $\sim 1.5 \%$ precision might be reachable using the $t \bar{b}$ decay mode [16]. The $\tau \nu_{\tau}$ decay mode, on the other hand, could yield a precision of $\sim 0.5 \%$ [79].

Due to the large number of MSSM parameters, certain benchmark scenarios $[8,81,82]$ (for real parameters) have been used for the interpretation of MSSM Higgs boson searches at LEP, the Tevatron and the LHC. Since at tree level the Higgs sector of the MSSM is governed by two parameters (in addition to the gauge couplings), the definition of the benchmarks is usually such that the two treelevel parameters, $M_{A}$ and $\tan \beta$, are varied while the values of all other parameters are fixed at certain benchmark settings. The most commonly used benchmark scenario for the $C P$-conserving MSSM has been the $m_{h}^{\max }$ scenario $[8,81,82]$, and we therefore employ this scenario in our analysis. While the interpretation of the newly discovered Higgs-like state as the light MSSM Higgs boson is compatible with the $m_{h}^{\max }$ scenario only within a strip at relatively low $\tan \beta$, it should be noted that changing the stop mixing parameter $X_{t}$ from the "maximal" value of $X_{t} / M_{\text {SUSY }} \sim 2$ to slightly smaller values (with the other parameters fixed) yields $M_{h} \sim 126 \mathrm{GeV}$ over large parts of the parameter space, see Ref. [8]. Consequently, this scenario is expected to provide a good indication of the possible size of the radiative corrections to $M_{H^{ \pm}}$. The scenario is defined as follows:

The $m_{h}^{\max }$ scenario:

In this scenario the parameters are chosen such that the mass of the light $C P$-even Higgs boson acquires its maximum possible values as a function of $\tan \beta$ (for fixed $M_{\text {SUSY }}, m_{t}$ and $M_{A}$ set to its maximum value, $M_{A}=1 \mathrm{TeV}$ ). This was used in particular to obtain conservative $\tan \beta$ exclusion bounds [83] at LEP [11]. The parameters are (including the most recent value for $m_{t}$ [84])

$$
\begin{aligned}
m_{t} & =\mu^{\overline{\mathrm{DR}}}=173.2 \mathrm{GeV}, \\
M_{\mathrm{SUSY}} & =1 \mathrm{TeV} \\
\mu & =200 \mathrm{GeV}, \\
M_{2} & =200 \mathrm{GeV}, \\
X_{t} & =2 M_{\mathrm{SUSY}} \\
A_{b} & =A_{t} \\
m_{\tilde{g}} & =0.8 M_{\text {SUSY }} .
\end{aligned}
$$

SUSY-breaking parameters in the $\tilde{t} / \tilde{b}$ mass matrices, see Eq. (20), that are all chosen to be equal. $M_{\text {SUSY }}$ and $X_{t}$ in this scenario correspond to the parameters used to express the $m_{t}^{4} / M_{W}^{2}$ corrections as given in Eqs. (50) and (53). In order to avoid conflicts with the LHC searches for squarks of the first and second generation, contrary to the original definition [81], $M_{\text {SUSY }}$ should only be considered to fix the soft SUSY-breaking parameters for the squarks of the third generation, while the first two generations play a small role for the MSSM Higgs phenomenology. To fix a value for the squarks of the first two generations, for sake of simplicity, we kept the value of $M_{\text {SUSY }}$, but choosing higher values has a minor impact (see below). The gluino mass parameter, $m_{\tilde{g}}$, might also be in conflict with recent LHC SUSY searches. However, since also the impact of $m_{\tilde{g}}$ is relatively small, we keep its value at the original definition. (A slightly higher value is chosen in the updated version of this scenario in Ref. [8].)

As discussed above, there are also potentially large corrections in the $b / \tilde{b}$ sector, depending on the value and sign of the parameter $\mu$ [82]. Consequently, besides analyzing the $M_{H^{ \pm}}$dependence on $M_{A}$ and $\tan \beta$, we also study the effect of a variation of $\mu$, allowing both an enhancement and a suppression of the bottom Yukawa coupling. Concerning the $m_{h}^{\max }$ benchmark scenario, as discussed in Refs. [82,85] (see also Ref. [86]), the $\Delta_{b}$ effects are particularly pronounced, since the two terms in Eq. (35) are of similar size.

The other MSSM parameters that are not specified above, such as the slepton masses, have only a minor impact on MSSM Higgs boson phenomenology. In our numerical analysis below we fix them such that all soft SUSY-breaking parameters in the diagonal entries of the slepton mass matrices are set to $M_{\mathrm{SUSY}}$, and the trilinear couplings for all sfermions are set to $A_{t}$, if not indicated differently for $A_{b}\left(=A_{\tau}\right)$.

For the analysis of the size of the two-loop corrections we employ in addition also a scenario that yields particularly interesting phenomenology for the charged Higgs boson. In this scenario the heavy $C P$-even Higgs boson is interpreted as the newly discovered particle at $\sim 126 \mathrm{GeV}$, see, e.g., Refs. [5-9]. The starting point for this scenario is the "best-fit" value obtained in a seven parameter fit in the MSSM, where the interpretation of the signal at $\sim 126 \mathrm{GeV}$ as the heavy $C P$-even Higgs boson of the MSSM has been confronted with the measured signal strengths, taking into account also constraints from electroweak precision observables and flavor physics [7]. The parameters are (close to the parameters in the "low- $M_{H}$ scenario defined in Ref. [8])"

The light heavy-Higgs scenario: 


$$
\begin{aligned}
m_{t} & =173.2 \mathrm{GeV}, \\
M_{\tilde{q}_{3}} & =M_{\tilde{t}_{L}}\left(=M_{\tilde{b}_{L}}\right)=M_{\tilde{t}_{R}}=M_{\tilde{b}_{R}}=670 \mathrm{GeV}, \\
M_{\tilde{l}_{3}} & =M_{\tilde{\tau}_{L}}\left(=M_{\tilde{\nu}_{L}}\right)=M_{\tilde{\tau}_{R}}=323 \mathrm{GeV}, \\
A_{f} & =1668 \mathrm{GeV}, \\
M_{A} & =124.2 \mathrm{GeV}, \\
\tan \beta & =9.8, \quad \mu=2120 \mathrm{GeV}, \\
M_{2} & =304 \mathrm{GeV}, \\
M_{\tilde{q}_{L}} & =M_{\tilde{q}_{R}}(q=c, s, u, d)=1000 \mathrm{GeV}, \\
M_{\tilde{l}_{L}} & =M_{\tilde{l}_{R}}\left(l=\mu, \nu_{\mu}, e, \nu_{e}\right)=300 \mathrm{GeV}, \\
m_{\tilde{g}} & =1000 \mathrm{GeV}, \\
M_{1} & =\frac{5}{3} \frac{s_{\mathrm{w}}^{2}}{c_{\mathrm{w}}^{2}} M_{2} \approx \frac{1}{2} M_{2},
\end{aligned}
$$

where the latter four were fixed in the fit. $M_{\tilde{q}_{3}}$ denotes the diagonal soft SUSY-breaking parameter for the third generation squarks, $M_{\tilde{q}_{L}}$ and $M_{\tilde{q}_{R}}$ for the first and second generation squarks, $M_{\tilde{l}_{3}}$ for the third generation sleptons, and $M_{\tilde{l}_{L}}$ and $M_{\tilde{l}_{R}}$ for the first and second generation sleptons. $A_{f}$ denotes the trilinear Higgs-sfermion coupling which is taken to be equal for all sfermions.

\section{A. One-loop corrections}

We start with the analysis of the various one-loop contributions. In Figs. 5 and 6 we show $\Delta M_{H^{ \pm}}:=M_{H^{ \pm}}-$ $m_{H^{ \pm}}$, i.e. the difference between the result with radiative

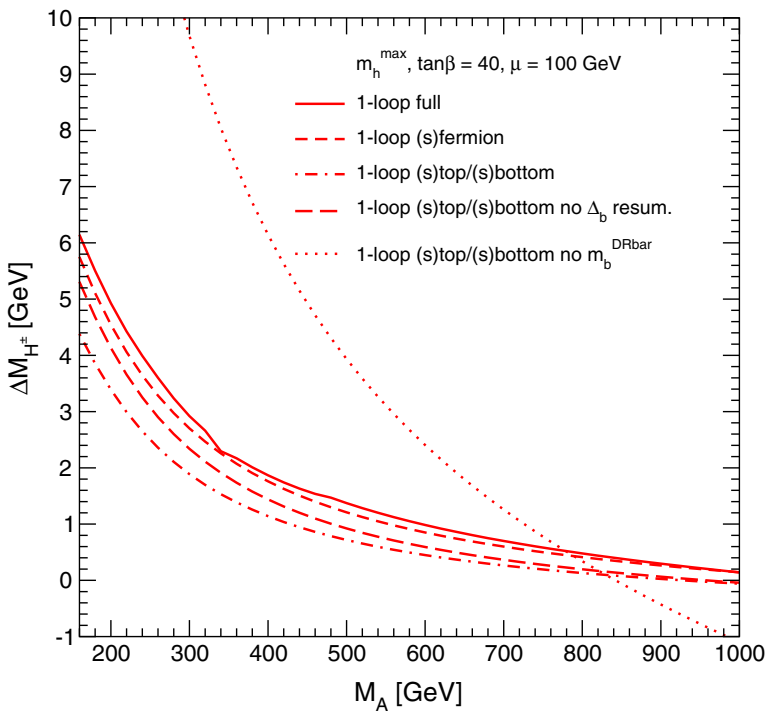

corrections and the tree-level value, in various approximations. The solid lines are the full one-loop result including the $\Delta_{b}$ resummation, see Eq. (35). The first approximation to this is shown as short-dashed lines, where only the contributions from SM fermions and their SUSY partners (i.e. all squarks and sleptons) are taken into account, still including the $\Delta_{b}$ corrections. The next step of approximation is shown as dot-dashed lines, where only corrections from the $t / b$ and $\tilde{t} / \tilde{b}$ sector are included, still with the $\Delta_{b}$ resummation. The penultimate step of the approximation is to leave out the $\Delta_{b}$ corrections, but using $\bar{m}_{b}$ [i.e. including the SM QCD corrections, see Eq. (33)] in the Higgs boson couplings, shown as the long-dashed lines. The final step in the approximation is to drop the SM QCD corrections, i.e. replacing $\bar{m}_{b}$ by the bottom pole mass, $m_{b}=4.8 \mathrm{GeV}$, in the Higgs Yukawa couplings, shown as the dotted lines.

First, in Fig. 5, we analyze the dependence of $M_{H^{ \pm}}$on $M_{A}$ in the $m_{h}^{\max }$ scenario. The left (right) plot of Fig. 5 shows $\Delta M_{H^{ \pm}}$as a function of $M_{A}$ for $\tan \beta=40$ and $\mu=100(1000) \mathrm{GeV}$. It should be noted that the very low $M_{A}$ values are by now ruled out by the LHC heavy MSSM Higgs boson searches [12] for this value of $\tan \beta$. However, in order to display the full parameter dependence we do not include these bounds here. The full result (solid lines) yields one-loop corrections between 1.5 and $6.0 \mathrm{GeV}$ for low $M_{A}$, becoming smaller for increasing $M_{A}$. The still allowed $M_{A}$ values should give one-loop corrections of $\mathcal{O}(2 \mathrm{GeV})$ in this scenario for small $\mu$. The $f / \tilde{f}$ sector (short-dashed) gives a rather good approximation, better than $0.5 \mathrm{GeV}$. Going to the $t / b / \tilde{t} / \tilde{b}$ approximations (dotdashed) yields a prediction that differs from the full result

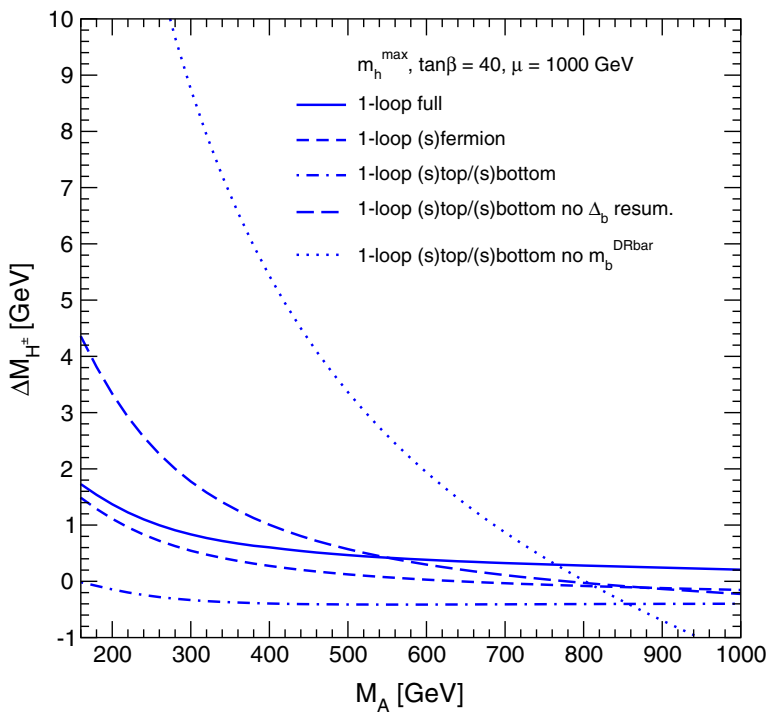

FIG. 5 (color online). $\Delta M_{H^{ \pm}}:=M_{H^{ \pm}}-m_{H^{ \pm}}$is shown in the $m_{h}^{\max }$ scenario as a function of $M_{A}$ for $\mu=100 \mathrm{GeV}$ (left) and $\mu=1000 \mathrm{GeV}$ (right) and $\tan \beta=40$, evaluated at the one-loop level. We show the full one-loop result including $\Delta_{b}$ corrections (solid lines), the pure SM fermion/sfermion contribution (short-dashed), the $\tilde{t} / \tilde{b}$ contribution (dot-dashed), the $\tilde{t} / \tilde{b}$ corrections excluding the $\Delta_{b}$ corrections but using $\bar{m}_{b}$ (long-dashed), and the $\tilde{t} / \tilde{b}$ corrections excluding the $\Delta_{b}$ resummation and using the bottom pole mass, $m_{b}$ (dotted). 

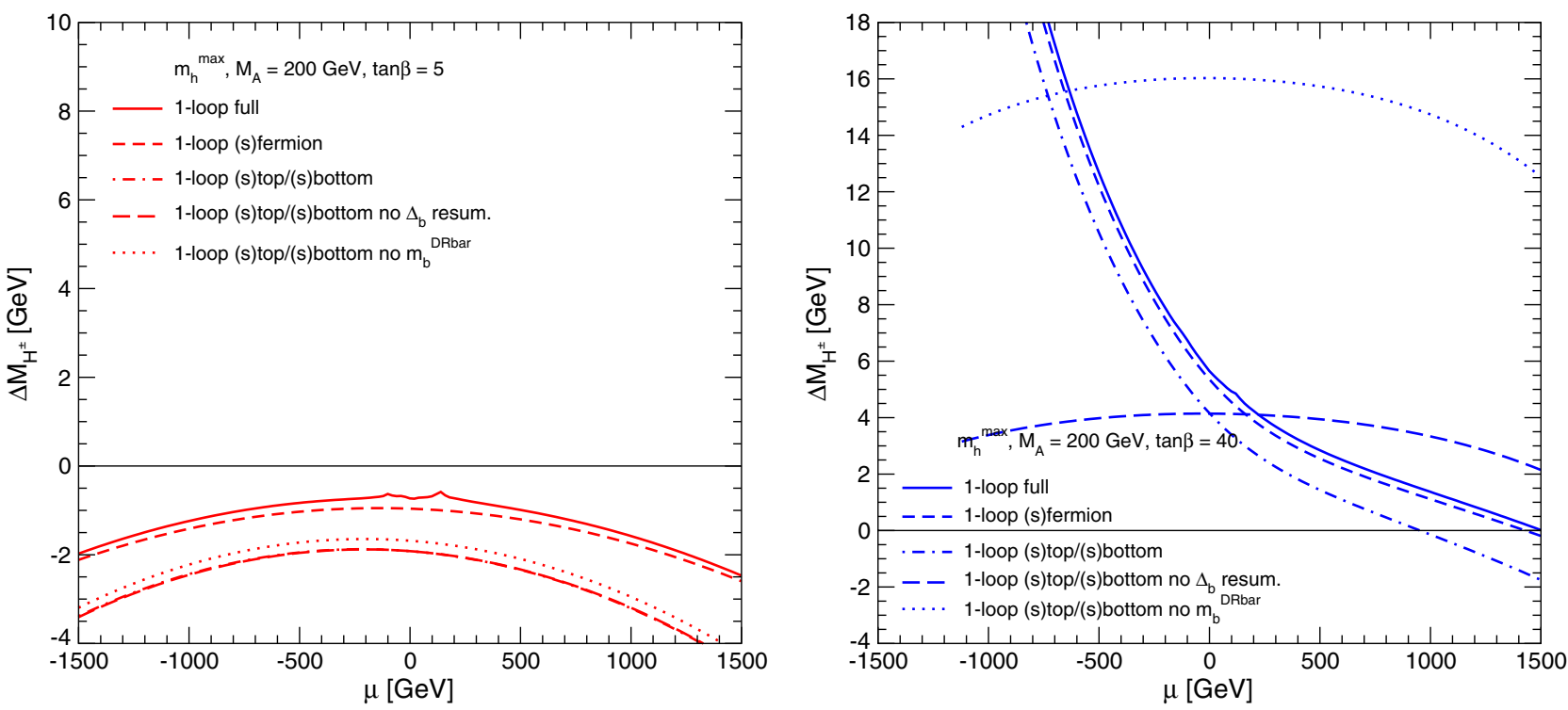

FIG. 6 (color online). $\Delta M_{H^{ \pm}}:=M_{H^{ \pm}}-m_{H^{ \pm}}$is shown in the $m_{h}^{\max }$ scenario as a function of $\mu$ for $\tan \beta=5$ (left) and tan $\beta=40$ (right) and $M_{A}=200 \mathrm{GeV}$, evaluated at the one-loop level. The line coding is as in Fig. 5.

by up to $\sim 2 \mathrm{GeV}$ for low $M_{A}$. The $f / \tilde{f}$ corrections besides the ones from third generation squarks are roughly independent of the Yukawa couplings of the various (s)fermions and are of pure electroweak type, and can grow as $\log \left(M_{\text {SUSY }} / M_{W}\right)$ [62], and larger masses lead to larger corrections. Consequently, taking into account only the third generation (s)quark contribution can yield nonnegligible uncertainties in the $M_{H^{ \pm}}$prediction. In the next step the $\Delta_{b}$ corrections are neglected, which are formally beyond the one-loop order, resulting in the long-dashed lines. The comparison between the dot-dashed and the long-dashed lines shows that the impact of the $\Delta_{b}$ corrections is small, below $\sim 500 \mathrm{MeV}$ for $\mu=100 \mathrm{GeV}$, but can be larger than $4 \mathrm{GeV}$ for $\mu=1000 \mathrm{GeV}$, see Eq. (35). Finally we consider an approximation where the SM QCD corrections to the bottom Yukawa coupling are dropped, i.e. $m_{b}$ is used instead of $\bar{m}_{b}$, resulting in the dotted lines. These contributions can be larger than all other steps of approximations in the region of large $\tan \beta$ considered here. Neglecting the SM QCD corrections in $m_{b}$ shifts $\Delta M_{H^{ \pm}}$upwards by more than $10 \mathrm{GeV}$, depending on the scenario.

In order to analyze the dependence of the $M_{H^{ \pm}}$prediction on $\mu$ we show in Fig. $6 \Delta M_{H^{ \pm}}$in the $m_{h}^{\max }$ scenario as a function of $\mu$ for $M_{A}=200 \mathrm{GeV}$ and $\tan \beta=5(40)$ in the left (right) plot. Again, the large $\tan \beta$ values are by now experimentally excluded by the LHC heavy MSSM Higgs searches for this value of $M_{A}$ [12], but the two "extreme" $\tan \beta$ values are meant to give an idea about the possible variations. We start with the case of $\tan \beta=5$, see the left plot of Fig. 6 . The $t / b / \tilde{t} / \tilde{b}$ corrections neglecting the SM QCD corrections (dotted line) are nearly symmetric in $\mu$, ranging between -2 and $-4 \mathrm{GeV}$.
Including the SM QCD corrections (long-dashed) has a negligible impact. The same holds for the $\Delta_{b}$ corrections (dashed-dotted) due to the small value of $\tan \beta$, and the two lines lie on top of each other. Including the full (s)fermion corrections, on the other hand, has a sizable impact on the result. The contributions from the other (s)fermions partially cancel the $t / b / \tilde{t} / \tilde{b}$ corrections. Including also the non-(s)fermionic contributions yields a total one-loop effect that stays below $\sim-2 \mathrm{GeV}$.

The results look quite different for $\tan \beta=40$ as shown in the right plot of Fig. 6. For negative $\mu$, the enhancement of the bottom Yukawa coupling can become very strong due to the large $\tan \beta$ value. In the $m_{h}^{\max }$ scenario $\mu \lesssim$ $-1200 \mathrm{GeV}$ yields $\Delta_{b} \rightarrow-1$, i.e. the model enters the nonperturbative regime, and no evaluations in the Higgs sector are possible. Consequently, the corresponding curves in the right plot of Fig. 6 stop at $\mu \approx-1100 \mathrm{GeV}$. The pure $t / b / \tilde{t} / \tilde{b}$ corrections (dotted line) reach $13-16 \mathrm{GeV}$ if they are evaluated with the bottom pole mass. Including the SM QCD corrections (long-dashed) into the effective bottom-quark mass strongly reduces the effect to the level of $2-4 \mathrm{GeV}$. In the next step the $\Delta_{b}$ effects are included (dot-dashed line). Due to $\Delta_{b} \propto \mu \tan \beta$ the inclusion of $\Delta_{b}$ results in a strong asymmetry of $\Delta M_{H^{ \pm}}$with a larger correction to $M_{H^{ \pm}}$for negative $\mu$ (corresponding to an enhanced bottom Yukawa coupling) and a much smaller correction for positive $\mu$ (corresponding to a suppressed bottom Yukawa coupling). Including the full one-loop corrections the overall correction in the $m_{h}^{\max }$ scenario ranges from $\Delta M_{H^{ \pm}} \geqslant 18 \mathrm{GeV}$ for $\mu \lesssim-1000 \mathrm{GeV}$ to $\Delta M_{H^{ \pm}} \approx 0$ for $\mu=+1500 \mathrm{GeV}$.

The dependence on $\tan \beta$ is analyzed in Fig. 7. We show $\Delta M_{H^{ \pm}}$in the $m_{h}^{\max }$ scenario as a function of $\tan \beta$ for 

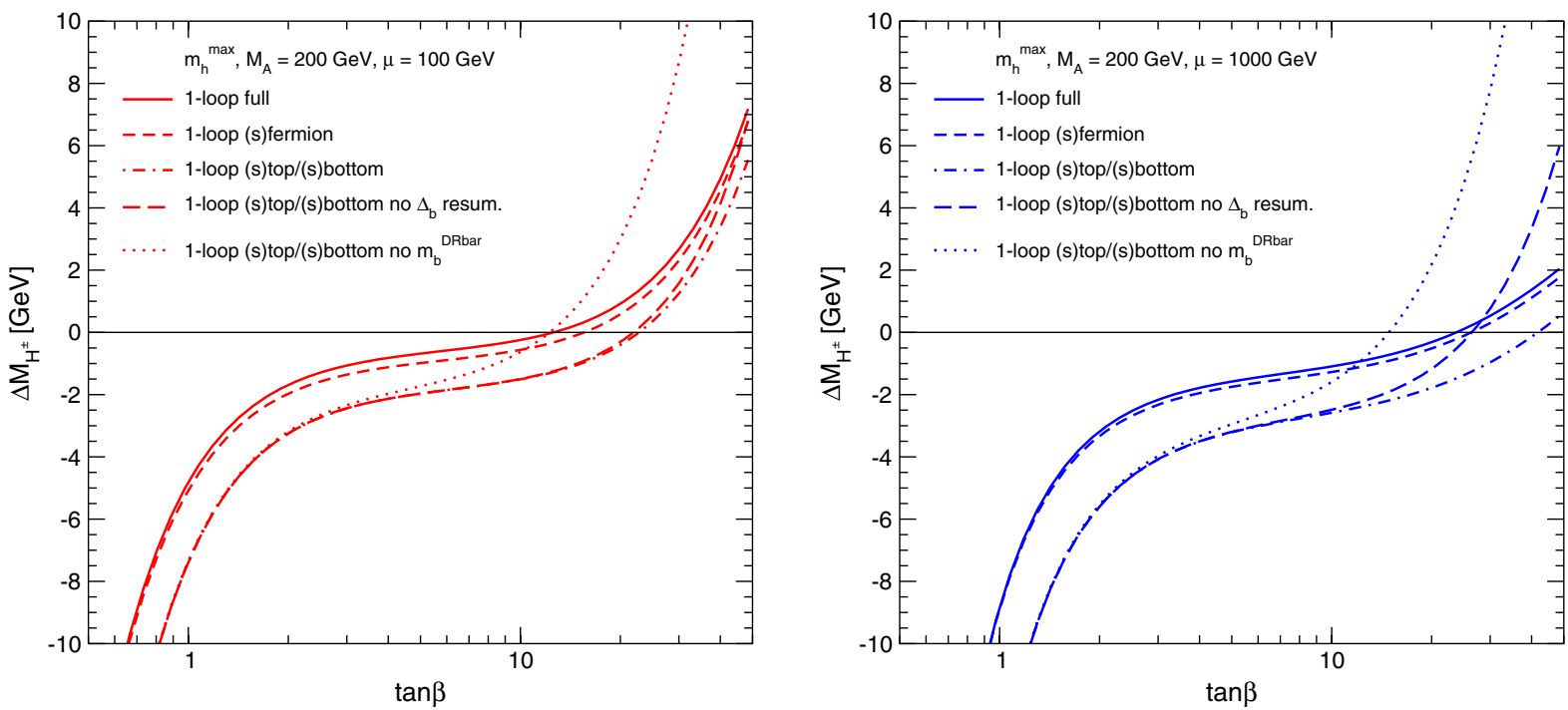

FIG. 7 (color online). $\Delta M_{H^{ \pm}}:=M_{H^{ \pm}}-m_{H^{ \pm}}$is shown in the $m_{h}^{\max }$ scenario as a function of $\tan \beta$ for $\mu=100 \mathrm{GeV}$ (left) and $\mu=1000 \mathrm{GeV}$ (right) and $M_{A}=200 \mathrm{GeV}$, evaluated at the one-loop level. The line coding is as in Fig. 5.

$M_{A}=200 \mathrm{GeV}$ and as before for $\mu=100(1000) \mathrm{GeV}$ in the left (right) plot. It should be noted that values of $\tan \beta$ around 1 are excluded by LEP Higgs searches [11], whereas large values are excluded by LHC Higgs searches for this value of $M_{A}$ [12]. The sign and size of the one-loop correction to $M_{H^{ \pm}}$depends strongly on $\tan \beta$, which enters the Higgs couplings to (s)fermions as well as the $\Delta_{b}$ corrections. Negative corrections occur for $\tan \beta \lesssim 10$, while positive values of $\Delta M_{H^{ \pm}}$are obtained for large $\tan \beta$ values. In the phenomenologically allowed region of $\tan \beta$ the corrections stay within $\Delta M_{H^{ \pm}}= \pm 2 \mathrm{GeV}$. As in the plots of Fig. 5, the effect of the non-sfermion sector in comparison with the $f / \tilde{f}$ contributions (short-dashed lines) is relatively small and stays below $0.5 \mathrm{GeV}$.

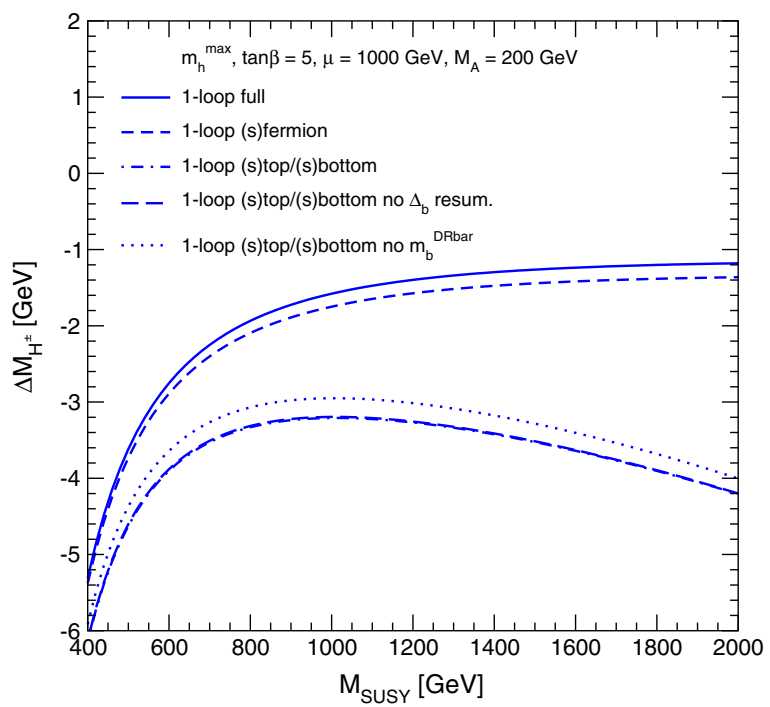

The Yukawa coupling independent effects (dot-dashed lines) are $\sim 2 \mathrm{GeV}$, largely independent of $\tan \beta$. The contribution from the $\Delta_{b}$ effects is negligible for $\tan \beta \lesssim 5$ and grows with increasing $\tan \beta$, reaching several $\mathrm{GeV}$ for large $\tan \beta$ and $\mu=1000 \mathrm{GeV}$. On the other hand, for $\mu=100 \mathrm{GeV}$ these corrections stay very small even for the largest $\tan \beta$ values. The biggest effects again can arise from the inclusion of the SM QCD corrections to $m_{b}$ for $\tan \beta \geqslant 5$. Largely independently of the scenario and the choice for $\mu$ they reach $5-10 \mathrm{GeV}$.

Next, in Fig. 8 we show the dependence on $M_{\text {SUSY }}$. The SUSY mass scale (which we chose to be equal for all sfermions, see above) enters via contributions $\propto \log \left(M_{\mathrm{SUSY}} / M_{W}\right)$ or $\propto M_{W}^{2} / M_{\mathrm{SUSY}}^{2}$ into the charged

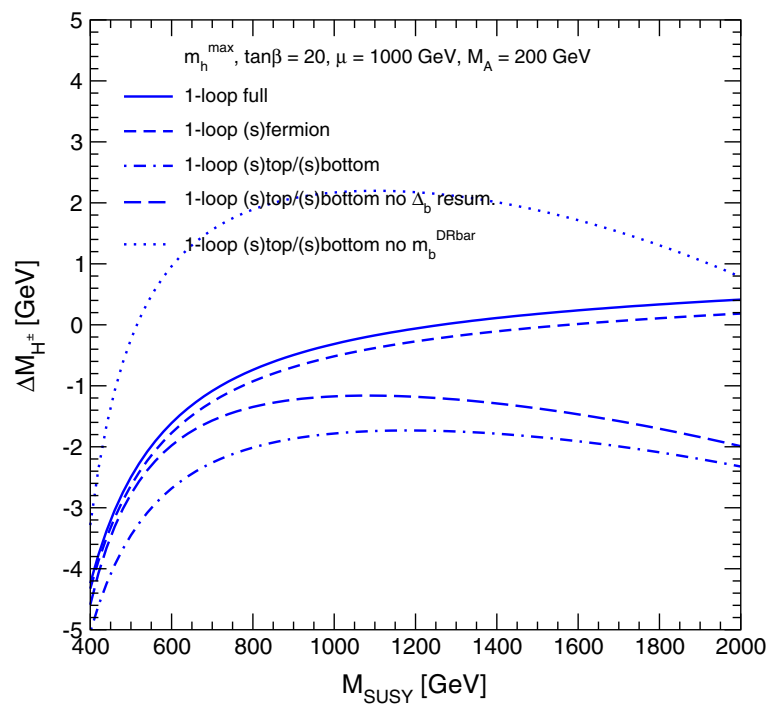

FIG. 8 (color online). $\Delta M_{H^{ \pm}}:=M_{H^{ \pm}}-m_{H^{ \pm}}$is shown in the $m_{h}^{\max }$ scenario as a function of $M_{\mathrm{SUSY}}$ for tan $\beta=5$ (left) and $\tan \beta=20$ (right), $M_{A}=200 \mathrm{GeV}$ and $\mu=1000 \mathrm{GeV}$, evaluated at the one-loop level. The line coding is as in Fig. 5 . 
Higgs boson mass prediction [62], where several competing contributions have been identified. One is proportional to large Yukawa couplings from the top/bottom sector, while another one stems from the electroweak couplings of scalar fermions and is similar for all flavors.

In the left plot of Fig. 8 we show $\Delta M_{H^{ \pm}}$as a function of $M_{\text {SUSY }}$ in the $m_{h}^{\max }$ scenario for $\mu=1000 \mathrm{GeV}, M_{A}=$ $200 \mathrm{GeV}$ and $\tan \beta=5$. One can see that the $b / \tilde{b}$ contributions, which are influenced strongly by the bottom Yukawa coupling and the $\Delta_{b}$ corrections, do not play a prominent role as they change $\Delta M_{H^{ \pm}}$only weakly for small $\tan \beta$. The contributions from the lighter fermions (i.e. neither top nor bottom) and their SUSY partners, on the other hand, become very important for $M_{\text {SUSY }} \gtrsim$ $1000 \mathrm{GeV}$. Without those corrections (dot-dashed line) rather large negative contributions to $M_{H^{ \pm}}$would occur for large $M_{\mathrm{SUSY}}$, while including these corrections (shortdashed line) $\Delta M_{H^{ \pm}}$flattens out for large $M_{\text {SUSY }}$, reaching $\sim-1 \mathrm{GeV}$ at $M_{\mathrm{SUSY}}=2000 \mathrm{GeV}$. The corrections from the non-(s)fermion sector are small and change $M_{H^{ \pm}}$by less than about $0.2 \mathrm{GeV}$. A qualitatively similar behavior can be observed for $\tan \beta=20$ (which is close to the current sensitivity limits of heavy MSSM Higgs searches at the LHC [12]) as shown in the right plot of Fig. 8. Due to the larger value of $\tan \beta$ the $b / \tilde{b}$ corrections and $\Delta_{b}$ effects are much more pronounced. The contributions from the (s)fermion sector beyond $t / \tilde{t} / b / \tilde{b}$ are sizable for $M_{\text {SUSY }} \gtrsim$ $1000 \mathrm{GeV}$. Due to numerical cancellations the full oneloop correction to $M_{H^{ \pm}}$is close to zero for this part of the SUSY parameter space. This is in agreement with the right plot of Fig. 7. In summary, for large $M_{\text {SUSY }}$ especially the corrections from the full (s)fermion sector have to be taken into account.

We finally analyze the size of the full one-loop corrections in the case of $A_{t} \neq A_{b}$ in Fig. 9. We show the results in the $A_{b}-A_{t}$ plane for $M_{A}=200(120) \mathrm{GeV}$ in the top (bottom) row and $\tan \beta=40$ (10) in the left (right) column. Again, the extreme choices for $M_{A}$ and $\tan \beta$, partially excluded by LHC Higgs searches [12] indicate the range of the possible size of the corrections. The other parameters are $M_{\text {SUSY }}=500 \mathrm{GeV}, \mu=1000 \mathrm{GeV}, \quad M_{2}=$ $500 \mathrm{GeV}$.

The color coding in the plots tells the value of $\Delta M_{H^{ \pm}}$. At small $\tan \beta$, as can be seen in the upper right plot, the value of $\Delta M_{H^{ \pm}}$depends mainly on $A_{t}$, i.e. the convention $A_{b}=A_{t}$ in the $m_{h}^{\max }$ scenario does not have a relevant impact on the $M_{H^{ \pm}}$evaluation at the one-loop level for small $\tan \beta$. This changes for large $\tan \beta$ as can be
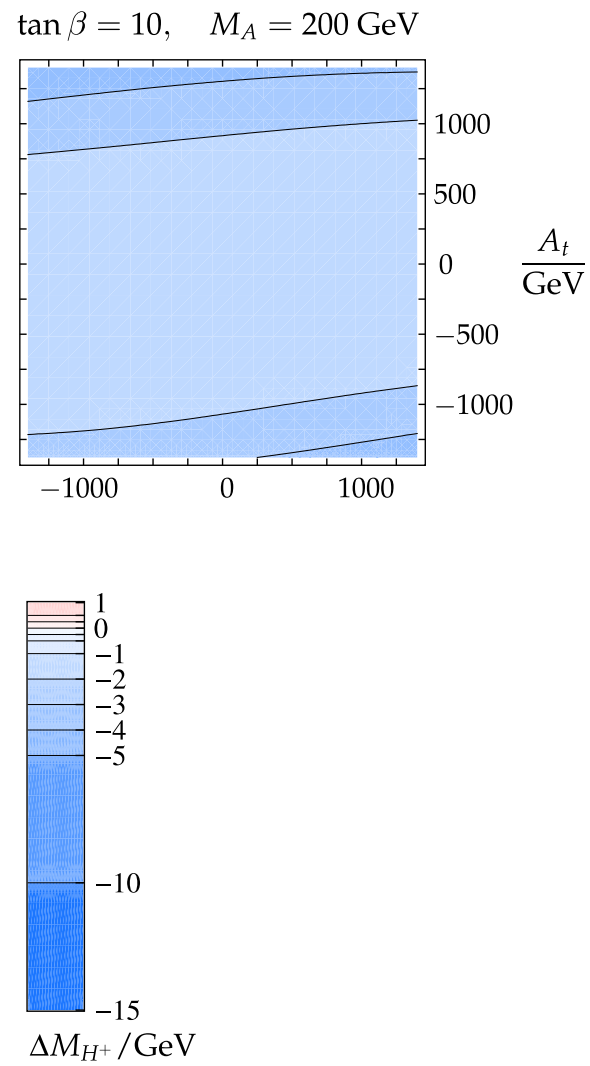

FIG. 9 (color online). The size of the full one-loop correction $\Delta M_{H^{ \pm}}:=M_{H^{ \pm}}-m_{H^{ \pm}}$is shown in the $A_{b}-A_{t}$ plane for $M_{A}=200(120) \mathrm{GeV}$ in the top (bottom) row and $\tan \beta=40(10)$ in the left (right) column. The other parameters are $M_{\text {SUSY }}=500 \mathrm{GeV}, \mu=1000 \mathrm{GeV}, M_{2}=500 \mathrm{GeV}$. 
observed in the two left plots of the figure. The main diagonal corresponds to $A_{t}=A_{b}$ and exhibits (for both $M_{A}$ values) relatively small corrections up to $\sim 3 \mathrm{GeV}$. The other extreme, $A_{t}=-A_{b}$, on the other hand, yields much larger corrections, exceeding $\Delta M_{H^{ \pm}}=10 \mathrm{GeV}$ for large $\left|A_{t}\right|$. Consequently, a full one-loop calculation, allowing for different values of $A_{t}$ and $A_{b}$ is crucial for a precise $M_{H^{ \pm}}$evaluation.

\section{B. Two-loop corrections}

We now turn to the analysis of the effects of the two-loop corrections of $\mathcal{O}\left(\alpha_{t} \alpha_{s}\right)$, where in the plots we denote " 2 loop" as the full one-loop corrections supplemented by the $\mathcal{O}\left(\alpha_{t} \alpha_{s}\right)$ contributions. As described in Sec. II we derived the $\mathcal{O}\left(\alpha_{s}\right)$ corrections to the one-loop $\mathcal{O}\left(m_{t}^{4} / M_{W}^{2}\right)$ term. In our numerical analysis we concentrate on cases where on the one hand the full one-loop contribution to $M_{H^{ \pm}}$is sizable, and on the other hand the $\mathcal{O}\left(m_{t}^{4} / M_{W}^{2}\right)$ corrections yield a relatively good approximation to the full one-loop result. For these cases it can be expected that the $\mathcal{O}\left(\alpha_{t} \alpha_{s}\right)$ two-loop corrections also constitute a substantial part of the full two-loop contributions.

We focus here on relatively low $\tan \beta$, since it is known that at large $\tan \beta$ the bottom/sbottom one-loop corrections are sizable (see the previous subsection) and the $\mathcal{O}\left(\alpha_{t} \alpha_{s}\right)$ terms cannot be expected to capture a leading piece of the two-loop contributions. As can be seen in Eqs. (51) and (50), the $\mathcal{O}\left(m_{t}^{4} / M_{W}^{2}\right)$ terms going $\sim \mu$ are enhanced with $\tan \beta$. Therefore we present the two-loop $\mathcal{O}\left(\alpha_{t} \alpha_{s}\right)$ corrections as a function of $\mu$. We furthermore set $M_{A}=$ $200 \mathrm{GeV}$, which allows relatively large absolute higherorder corrections. The chosen parameters are thus mostly in agreement with the LHC heavy MSSM Higgs searches (and we will not address this issue in the rest of this subsection).

In Fig. 10 we present $\Delta M_{H^{ \pm}}:=M_{H^{ \pm}}-m_{H^{ \pm}}$in the $m_{h}^{\max }$ scenario for $M_{A}=200 \mathrm{GeV}$ and $\tan \beta=5$ as a function of $\mu$ for $M_{\text {SUSY }}=500(1000) \mathrm{GeV}$ in the left (right) plot. $M_{H^{ \pm}}$is evaluated including the $\mathcal{O}\left(\alpha_{t} \alpha_{s}\right)$ corrections and shown as the blue/dark gray solid line. Also shown are the corresponding one-loop results of $\mathcal{O}\left(m_{t}^{4} / M_{W}^{2}\right)$ (dashed line), the full one-loop corrections (red/light gray solid line) and the difference between the two-loop and the full one-loop result (dot-dashed line). Starting with the left plot, where we have set $M_{\text {SUSY }}=$ $500 \mathrm{GeV}$, we find that the full one-loop corrections are well approximated by the $\mathcal{O}\left(m_{t}^{4} / M_{W}^{2}\right)$ term. As expected for $\tan \beta=5$, the $\Delta_{b}$ corrections do not play a prominent role and $\Delta M_{H^{ \pm}}$appears nearly symmetric for positive and negative $\mu$. The corresponding two-loop corrections modify the full one-loop result by up to $\sim 2 \mathrm{GeV}$ for $|\mu| \sim$ $1500 \mathrm{GeV}$, i.e. the $\mathcal{O}\left(\alpha_{t} \alpha_{s}\right)$ corrections can be sizable in this case. A similar behavior can be observed in the right plot of Fig. 10, where we have set $M_{\text {SUSY }}=1000 \mathrm{GeV}$ [i.e. as in the original definition of the $m_{h}^{\max }$ scenario, Eq. (54)]. As expected, the absolute corrections to $M_{H^{ \pm}}$ turn out to be smaller, see also Fig. 8, and the two-loop terms contribute up to $\sim 1 \mathrm{GeV}$ for $|\mu| \sim 1500 \mathrm{GeV}$ (where our plot stops).

For the remaining analysis we stick to the lower value of $M_{\mathrm{SUSY}}=500 \mathrm{GeV}$, but go to somewhat larger $\tan \beta$ values and investigate also lower values of $M_{A}$. In Fig. 11 we show $\Delta M_{H^{ \pm}}$for $\tan \beta=10$ and $M_{A}=120(200) \mathrm{GeV}$ in the left (right) plot. The results look qualitatively similar to the case of $\tan \beta=5$ : the $m_{t}^{4} / M_{W}^{2}$ approximation works well for the full one-loop result. The two-loop corrections
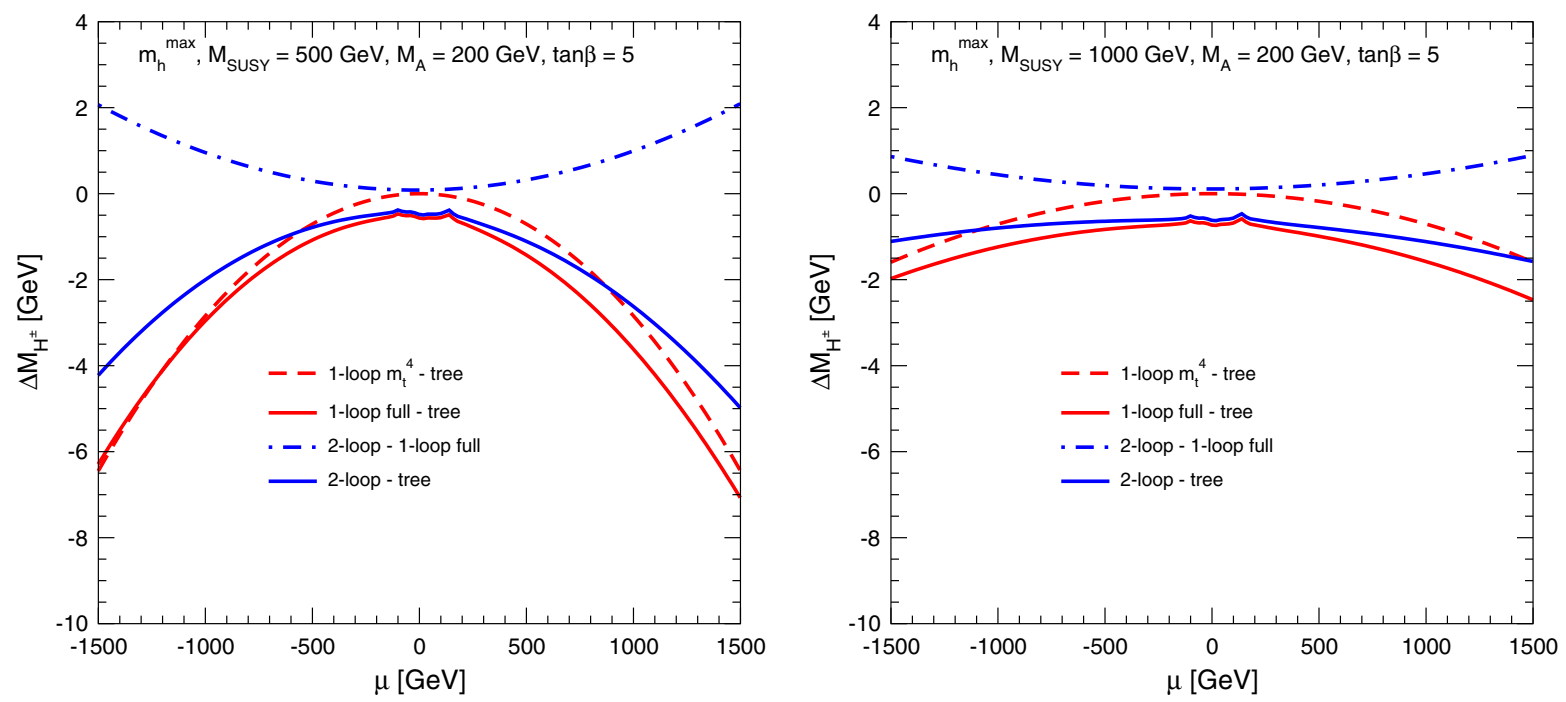

FIG. 10 (color online). $\Delta M_{H^{ \pm}}:=M_{H^{ \pm}}-m_{H^{ \pm}}$and $M_{H^{ \pm}}^{2 \text {-loop }}-M_{H^{ \pm}}^{1 \text {-loop }}$ are shown for $M_{A}=200 \mathrm{GeV}$ and tan $\beta=5$ as a function of $\mu$ in the $m_{h}^{\max }$ scenario. $M_{\text {SUSY }}$ is set to $500 \mathrm{GeV}$ (left) and to $1000 \mathrm{GeV}$ (right plot). $M_{H^{ \pm}}$is evaluated at the two-loop level (blue/dark gray solid). Also shown are the corresponding one-loop results of $\mathcal{O}\left(m_{t}^{4} / M_{W}^{2}\right)$ (dashed), the full one-loop corrections (red/light gray solid) and the difference between the two-loop and the full one-loop result (dot-dashed). 

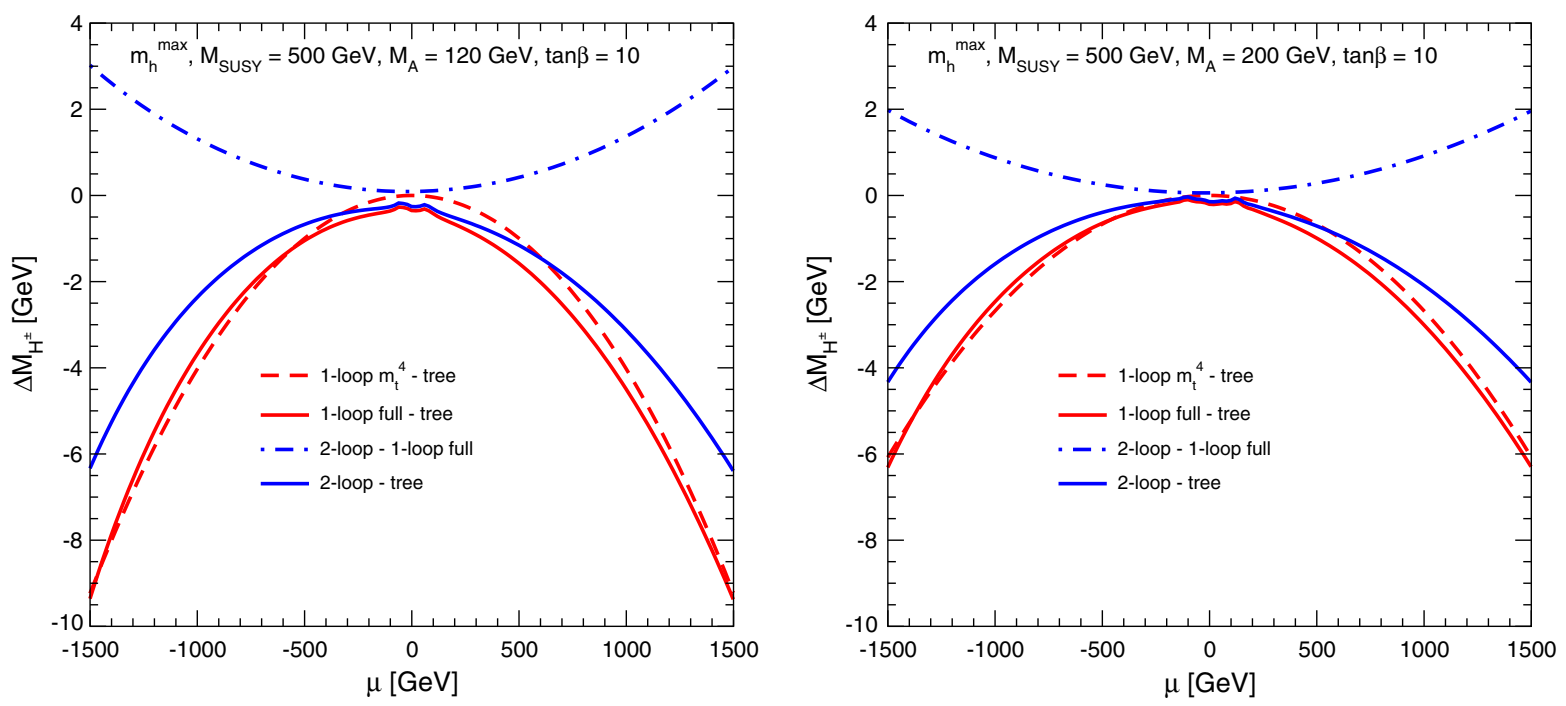

FIG. 11 (color online). $\Delta M_{H^{ \pm}}:=M_{H^{ \pm}}-m_{H^{ \pm}}$and $M_{H^{ \pm}}^{2 \text {-loop }}-M_{H^{ \pm}}^{1 \text {-loop }}$ are shown for $M_{A}=120 \mathrm{GeV}$ (left) and $M_{A}=200 \mathrm{GeV}$ (right plot), $\tan \beta=10$ and $M_{\text {SUSY }}=500 \mathrm{GeV}$ as a function of $\mu$ in the $m_{h}^{\max }$ scenario. $M_{H^{ \pm}}$is evaluated at the two-loop level (blue/ dark gray solid). Also shown are the corresponding one-loop results of $\mathcal{O}\left(m_{t}^{4} / M_{W}^{2}\right)$ (dashed), the full one-loop corrections (red/light gray solid) and the difference between the two-loop and the full one-loop result (dot-dashed).

go up to $\sim 3(2) \mathrm{GeV}$ for large values of $|\mu|$ for $M_{A}=$ 120(200) GeV.

In Fig. 12 we go to even higher $\tan \beta$ values and set $\tan \beta=20$, where $\Delta_{b}$ effects are expected to become relevant. As for the previous figure we fix $M_{A}=$ 120 (200) $\mathrm{GeV}$ in the left (right) plot. Sizable $\Delta_{b}$ effects can indeed be observed: for large negative values of $\mu$, $\mu \lesssim-1200 \mathrm{GeV}$ the $\Delta_{b}$ corrections become so large that an evaluation of the loop corrections to $M_{H^{ \pm}}$was not possible anymore (as was observed already in Fig. 6). For negative $\mu$ the $\mathcal{O}\left(m_{t}^{4} / M_{W}^{2}\right)$ corrections also start to deviate substantially from the full one-loop result, and the corresponding two-loop corrections cannot be expected to yield a good approximation to the full two-loop result in the region of relatively large negative $\mu$. For large and positive $\mu$, however, the $m_{t}^{4} / M_{W}^{2}$ approximation works very well both for $M_{A}=120 \mathrm{GeV}$ (left plot) and $M_{A}=$ $200 \mathrm{GeV}$ (right plot), so that in this region the $\mathcal{O}\left(\alpha_{t} \alpha_{s}\right)$ corrections can be expected to provide a reasonable approximation of the full two-loop corrections. For $\mu=+1500 \mathrm{GeV}$ the two-loop corrections again are sizable and amount up to $\sim 2-3 \mathrm{GeV}$.
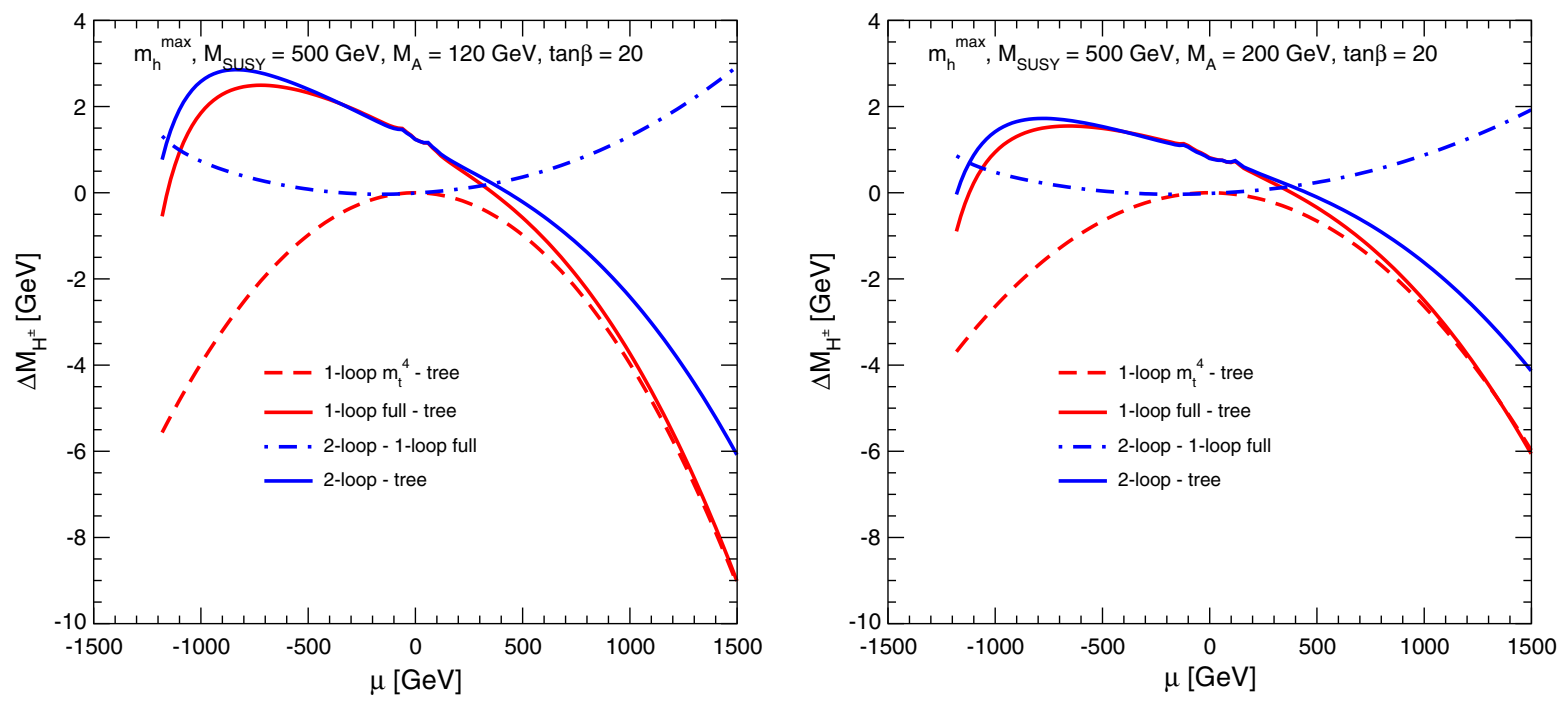

FIG. 12 (color online). $\Delta M_{H^{ \pm}}:=M_{H^{ \pm}}-m_{H^{ \pm}}$and $M_{H^{ \pm}}^{2 \text {-loop }}-M_{H^{ \pm}}^{1 \text {-loop }}$ are shown for $M_{A}=120 \mathrm{GeV}$ (left) and $M_{A}=200 \mathrm{GeV}$ (right plot), $\tan \beta=20$ and $M_{\text {SUSY }}=500 \mathrm{GeV}$ as a function of $\mu$ in the $m_{h}^{\max }$ scenario. $M_{H^{ \pm}}$is evaluated at the two-loop level (blue/ dark gray solid). Also shown are the corresponding one-loop results of $\mathcal{O}\left(m_{t}^{4} / M_{W}^{2}\right)$ (dashed), the full one-loop corrections (red/light gray solid) and the difference between the two-loop and the full one-loop result (dot-dashed). 

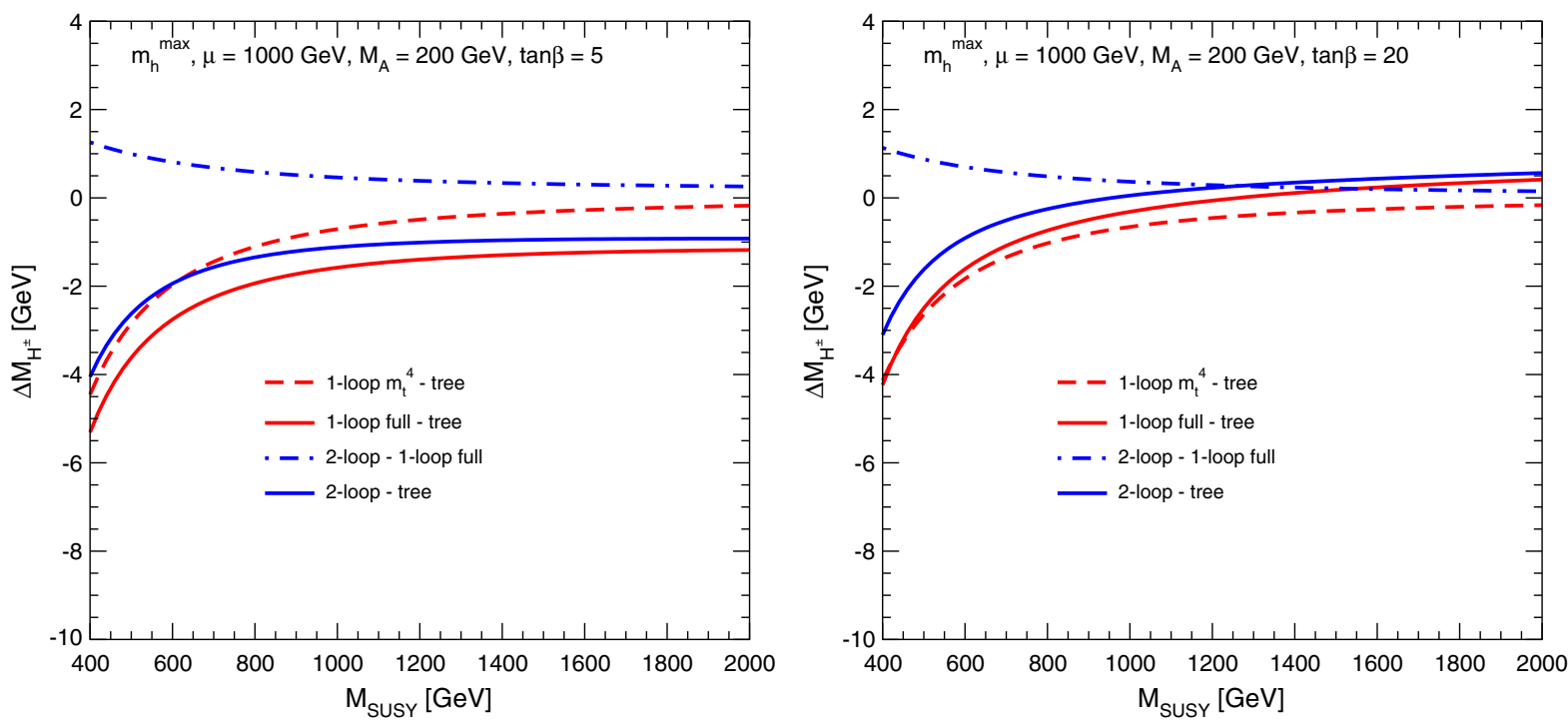

FIG. 13 (color online). $\Delta M_{H^{ \pm}}:=M_{H^{ \pm}}-m_{H^{ \pm}}$and $M_{H^{ \pm}}^{2 \text {-loop }}-M_{H^{ \pm}}^{1 \text {-loop }}$ are shown for $M_{A}=200 \mathrm{GeV}, \tan \beta=5$ (left) and $\tan \beta=20$ (right plot) and $\mu=1000 \mathrm{GeV}$ as a function of $M_{\mathrm{SUSY}}$ in the $m_{h}^{\max }$ scenario. $M_{H^{ \pm}}$is evaluated at the two-loop level (blue/dark gray solid). Also shown are the corresponding one-loop results of $\mathcal{O}\left(m_{t}^{4} / M_{W}^{2}\right)$ (dashed), the full one-loop corrections (red/light gray solid) and the difference between the two-loop and the full one-loop result (dot-dashed).

We complete our two-loop analysis in the $m_{h}^{\max }$ scenario with Fig. 13, where we show $\Delta M_{H^{ \pm}}$as a function of $M_{\text {SUSY }}$, in analogy to Fig. 8. In the left (right) plot we show the results for $\tan \beta=5(20)$ in the $m_{h}^{\max }$ scenario (i.e. $X_{t}=2 M_{\mathrm{SUSY}}$ and $m_{\tilde{g}}=0.8 M_{\mathrm{SUSY}}$ ) for $\mu=$ $1000 \mathrm{GeV}$ and $M_{A}=200 \mathrm{GeV}$. The $m_{t}^{4} / M_{W}^{2}$ corrections approximate the full one-loop results fairly well. The largest deviations occur for large values of $M_{\text {SUSY }}$, where the other (s)fermion sectors become more relevant, see the discussion on Fig. 8. For $M_{\mathrm{SUSY}}=400 \mathrm{GeV}$, the lowest value in our analysis, the two-loop $\mathcal{O}\left(\alpha_{t} \alpha_{s}\right)$ corrections amount up to $\sim 1 \mathrm{GeV}$. For large $M_{\text {SUSY }}$ this correction goes down nearly to zero.

Finally, in Fig. 14, we analyze the two-loop corrections to $M_{H^{ \pm}}$in the "light heavy-Higgs" scenario, in which the heavy $C P$-even Higgs boson is interpreted as the newly discovered particle at $\sim 126 \mathrm{GeV}$ [7]. We show the results as a function of $M_{A}$ (left) and $\tan \beta$ (right) with the other
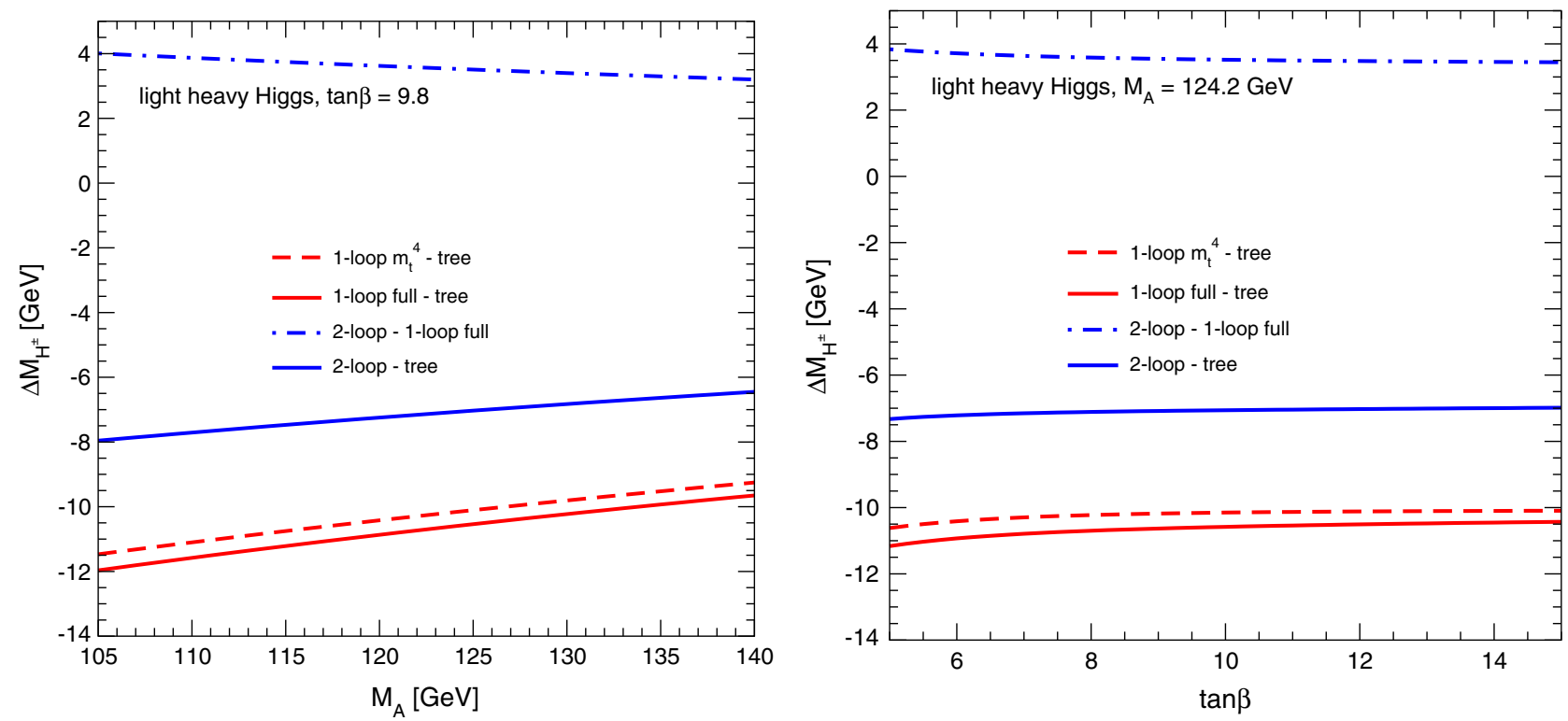

FIG. 14 (color online). $\Delta M_{H^{ \pm}}:=M_{H^{ \pm}}-m_{H^{ \pm}}$and $M_{H^{ \pm}}^{2 \text {-loop }}-M_{H^{ \pm}}^{1 \text {-loop }}$ are shown in the light heavy-Higgs scenario, as a function of $M_{A}$ with $\tan \beta=9.8$ (left) and as a function of $\tan \beta$ for $M_{A}=124.2 \mathrm{GeV}$ (right). $M_{H^{ \pm}}$is evaluated at the two-loop level (blue/dark gray solid). Also shown are the corresponding one-loop results of $\mathcal{O}\left(m_{t}^{4} / M_{W}^{2}\right)$ (dashed), the full one-loop corrections (red/light gray solid) and the difference between the two-loop and the full one-loop result (dot-dashed). 
parameters fixed as in Eq. (55). This scenario is characterized by a very rich phenomenology, since all five Higgs states in this case are rather light. Such a scenario can be probed at the LHC via searches for the heavier neutral Higgs bosons, $H$ and $A$, but also searches for a light charged Higgs boson that is produced in top-quark decays are of particular relevance in this case. As can be seen in Fig. 14 the $m_{t}^{4} / M_{W}^{2}$ corrections are an excellent approximation for the full one-loop result in the parameter space analyzed. The one-loop corrections are found to be large and negative in this case, while the two-loop corrections are positive and at the level of 3.5 to $4 \mathrm{GeV}$, amounting to about $30 \%$ of the one-loop corrections. Clearly, a thorough treatment of the higher-order contributions will be important for exploring the charged Higgs boson phenomenology in such a scenario.

\section{CONCLUSIONS}

We have presented a detailed analysis of the prediction for the charged Higgs boson mass, $M_{H^{ \pm}}$, within the MSSM, on the basis of a complete one-loop calculation, and incorporating the two-loop contributions of $\mathcal{O}\left(\alpha_{t} \alpha_{s}\right)$.

We find relatively large mass shifts at the one-loop level. In particular, we have analyzed the dependence of $M_{H^{ \pm}}$on the trilinear couplings $A_{t}$ and $A_{b}$. For the case $A_{t}=A_{b}$, which is assumed in the $m_{h}^{\max }$ benchmark scenario, corrections to $M_{H^{ \pm}}$of several $\mathrm{GeV}$ are found. The opposite case, $A_{t}=-A_{b}$, can yield much larger shifts exceeding $\Delta M_{H^{ \pm}}=10 \mathrm{GeV}$ for large $\left|A_{t}\right|$. In general, the full oneloop corrections are negative for small $\tan \beta$ and positive for large $\tan \beta$ in the $m_{h}^{\max }$ benchmark scenario.

Pronounced effects on $M_{H^{ \pm}}$in the region of large $\tan \beta$ originate from the standard QCD corrections to the bottom Yukawa coupling, formally a contribution beyond oneloop order. Similarly important are the shifts from the inclusion of $\Delta_{b}$ effects, leading to a strong dependence of $M_{H^{ \pm}}$on the size and the sign of $\mu$. The contributions from the (s)fermion sector beyond $t / \tilde{t} / b / \tilde{b}$ are sizable for $M_{\text {SUSY }} \gtrsim 1000 \mathrm{GeV}$ and can exceed $\sim 2 \mathrm{GeV}$.
The new two-loop corrections of $\mathcal{O}\left(\alpha_{t} \alpha_{s}\right)$ in most of the considered cases are of opposite sign to the one-loop corrections. The induced shifts in $M_{H^{ \pm}}$can be of several GeV for small $M_{A}$ and $\tan \beta$ and large values of $|\mu|$, and are thus of a size that may be probed at the LHC and the LC. The set of two-loop contributions considered here are expected to be particularly relevant for those MSSM parameter regions where the $m_{t}^{4} / M_{W}^{2}$ terms yield a good approximation to the full one-loop result, i.e. in particular for relatively low values of $\tan \beta$. For the general case, a more comprehensive higher-order calculation would be required.

In particular, we analyzed the size of the $\mathcal{O}\left(\alpha_{t} \alpha_{s}\right)$ corrections in the light heavy-Higgs scenario, in which the heavy $C P$-even Higgs boson is interpreted as the newly discovered particle at $\sim 126 \mathrm{GeV}$. In this scenario all MSSM Higgs bosons are relatively light, and there are interesting prospects for charged Higgs searches in topquark decays. The $m_{t}^{4} / M_{W}^{2}$ corrections yield an excellent approximation of the full one-loop result in this scenario. The genuine two-loop corrections are found to be up to $4 \mathrm{GeV}$, and thus are important for investigating charged Higgs phenomenology.

Our results for the charged Higgs boson mass are implemented into the public Fortran code FeynHiggs. The code also contains the evaluation of the charged Higgs boson decays and the main charged Higgs boson production channels at the LHC. The code can be obtained from http://www.feynhiggs.de.

\section{ACKNOWLEDGMENTS}

Work supported in part by the European Community's Marie-Curie Research Training Network under Contract No. MRTN-CT-2006-035505 "Tools and Precision Calculations for Physics Discoveries at Colliders." The work of S.H. was supported in part by CICYT (Grant No. FPA 2010-22163-C02-01) and by the Spanish MICINN's Consolider-Ingenio 2010 Program under Grant No. MultiDark CSD2009-00064. This work has been supported by the Collaborative Research Center SFB676 of the DFG, "Particles, Strings, and the Early Universe."
[1] G. Aad et al. (ATLAS Collaboration), Phys. Lett. B 716, 1 (2012).

[2] S. Chatrchyan et al. (CMS Collaboration), Phys. Lett. B 716, 30 (2012).

[3] M. Guillelmo Gomez-Ceballos et al. (CMS Collaboration), in Rencontres de Moriond EW 2013 (unpublished), https:// indico.in2p3.fr/getFile.py/access?contribId=16\&sessionId= $6 \&$ resId $=0 \&$ materialId $=$ slides\& $\operatorname{confId}=7411 ;$ F. Hubaut et al. (ATLAS Collaboration), in Rencontres de Moriond EW 2013 (unpublished), https://indico.in2p3.fr/getFile.py/ access? contribId $=45 \&$ sessionId $=6 \&$ res $I d=0 \&$ materialId $=$ slides \& confId $=7411$.

[4] H. Nilles, Phys. Rep. 110, 1 (1984); H. Haber and G. Kane, Phys. Rep. 117, 75 (1985); R. Barbieri, Riv. Nuovo Cimento 11N4, 1 (1988).

[5] S. Heinemeyer, O. Stål, and G. Weiglein, Phys. Lett. B 710, 201 (2012).

[6] R. Benbrik, M. Gomez Bock, S. Heinemeyer, O. Stål, G. Weiglein, and L. Zeune, Eur. Phys. J. C 72, 2171 (2012).

[7] P. Bechtle, S. Heinemeyer, O. Stål, T. Stefaniak, G. Weiglein, and L. Zeune, Eur. Phys. J. C 73, 2354 (2013). 
[8] M. Carena, S. Heinemeyer, O. Stål, C. Wagner, and G. Weiglein, arXiv:1302.7033.

[9] A. Bottino, N. Fornengo, and S. Scopel, Phys. Rev. D 85, 095013 (2012); M. Drees, Phys. Rev. D 86, 115018 (2012).

[10] R. Barate et al. (LEP Working Group for Higgs boson searches and ALEPH and DELPHI and L3 and OPAL Collaborations), Phys. Lett. B 565, 61 (2003).

[11] S. Schael et al. (ALEPH and DELPHI and L3 and OPAL and LEP Working Group for Higgs Boson Searches Collaborations), Eur. Phys. J. C 47, 547 (2006).

[12] S. Chatrchyan et al. (CMS Collaboration), Phys. Lett. B 713, 68 (2012); Report No. CMS-PAS-HIG-12-050.

[13] J. Aguilar-Saavedra et al., arXiv:hep-ph/0106315; see http://www.tesla.desy.de/tdr/; K. Ackermann et al., 4th ECFA/DESY Workshop on Physics and Detectors for a 90-GeV to 800-GeV Linear $e+e-$ Collider, Amsterdam, The Netherlands, 2003, Report No. DESY-PROC-2004-01.

[14] T. Abe et al. (American Linear Collider Working Group Collaboration), arXiv:hep-ex/0106056.

[15] K. Abe et al. (ACFA Linear Collider Working Group Collaboration), arXiv:hep-ph/0109166.

[16] S. Heinemeyer et al., arXiv:hep-ph/0511332.

[17] G. Weiglein et al. (LHC/ILC Study Group), Phys. Rep. 426, 47 (2006); A. De Roeck et al., Eur. Phys. J. C 66, 525 (2010).

[18] K. Desch, E. Gross, S. Heinemeyer, G. Weiglein, and L. Zivkovic, J. High Energy Phys. 09 (2004) 062.

[19] A. Heister et al. (ALEPH Collaboration), Phys. Lett. B 543, 1 (2002)

[20] J. Abdallah et al. (DELPHI Collaboration), Eur. Phys. J. C 34, 399 (2004).

[21] P. Achard et al. (L3 Collaboration), Phys. Lett. B 575, 208 (2003).

[22] D. Horváth et al. (OPAL Collaboration), Nucl. Phys. A721, C453 (2003); G. Abbiendi et al. (OPAL Collaboration), Eur. Phys. J. C 72, 2076 (2012).

[23] LEP Higgs Working Group for Higgs boson searches and ALEPH and DELPHI and L3 and OPAL Collaborations, arXiv:hep-ex/0107031.

[24] P. Lutz et al. (LEP Higgs Working Group), in Proceedings of the International Linear Collider Workshop (LCWS07 and ILC07), Hamburg, Germany, 2007, p. HIG04 (unpublished), http://www-zeuthen.desy.de/ILC/lcws07/LCWS07Higgs.htm.

[25] G. Abbiendi et al. (ALEPH and DELPHI and L3 and OPAL and The LEP working group for Higgs boson searches Collaborations), arXiv:1301.6065 [Eur. Phys. J. $\mathrm{C}$ (in preparation)].

[26] S. Chatrchyan et al. (CMS Collaboration), J. High Energy Phys. 07 (2012) 143.

[27] G. Aad et al. (ATLAS Collaboration), Eur. Phys. J. C 73, 2465 (2013).

[28] M. Frank, T. Hahn, S. Heinemeyer, W. Hollik, H. Rzehak, and G. Weiglein, J. High Energy Phys. 02 (2007) 047.

[29] S. Heinemeyer, W. Hollik, H. Rzehak, and G. Weiglein, Phys. Lett. B 652, 300 (2007).

[30] J. Ellis, G. Ridolfi, and F. Zwirner, Phys. Lett. B 257, 83 (1991); Y. Okada, M. Yamaguchi, and T. Yanagida, Prog. Theor. Phys. 85, 1 (1991); H. Haber and R. Hempfling, Phys. Rev. Lett. 66, 1815 (1991).
[31] A. Brignole, Phys. Lett. B 281, 284 (1992).

[32] P. Chankowski, S. Pokorski, and J. Rosiek, Phys. Lett. B 286, 307 (1992); Nucl. Phys. B423, 437 (1994).

[33] A. Dabelstein, Nucl. Phys. B456, 25 (1995); Z. Phys. C 67, 495 (1995).

[34] S. Heinemeyer, W. Hollik, and G. Weiglein, Phys. Rev. D 58, 091701 (1998); Phys. Lett. B 440, 296 (1998).

[35] S. Heinemeyer, W. Hollik, and G. Weiglein, Eur. Phys. J. C 9, 343 (1999).

[36] S. Heinemeyer, W. Hollik, and G. Weiglein, Phys. Lett. B 455, 179 (1999).

[37] S. Heinemeyer, W. Hollik, H. Rzehak, and G. Weiglein, Eur. Phys. J. C 39, 465 (2005).

[38] M. Carena, H. Haber, S. Heinemeyer, W. Hollik, C. Wagner, and G. Weiglein, Nucl. Phys. B580, 29 (2000).

[39] R. Zhang, Phys. Lett. B 447, 89 (1999); J. Espinosa and R. Zhang, J. High Energy Phys. 03 (2000) 026.

[40] G. Degrassi, P. Slavich, and F. Zwirner, Nucl. Phys. B611, 403 (2001).

[41] R. Hempfling and A. Hoang, Phys. Lett. B 331, 99 (1994).

[42] A. Brignole, G. Degrassi, P. Slavich, and F. Zwirner, Nucl. Phys. B631, 195 (2002).

[43] J. Espinosa and R. Zhang, Nucl. Phys. B586, 3 (2000).

[44] J. Espinosa and I. Navarro, Nucl. Phys. B615, 82 (2001).

[45] A. Brignole, G. Degrassi, P. Slavich, and F. Zwirner, Nucl. Phys. B643, 79 (2002).

[46] G. Degrassi, A. Dedes, and P. Slavich, Nucl. Phys. B672, 144 (2003).

[47] M. Carena, J. Espinosa, M. Quirós, and C. Wagner, Phys. Lett. B 355, 209 (1995); M. Carena, M. Quirós, and C. Wagner, Nucl. Phys. B461, 407 (1996).

[48] J. Casas, J. Espinosa, M. Quirós, and A. Riotto, Nucl. Phys. B436, 3 (1995); B439, 466(E) (1995).

[49] R. Hempfling, Phys. Rev. D 49, 6168 (1994); L. Hall, R. Rattazzi, and U. Sarid, Phys. Rev. D 50, 7048 (1994); M. Carena, M. Olechowski, S. Pokorski, and C. Wagner, Nucl. Phys. B426, 269 (1994).

[50] M. Carena, D. Garcia, U. Nierste, and C. Wagner, Nucl. Phys. B577, 88 (2000).

[51] H. Eberl, K. Hidaka, S. Kraml, W. Majerotto, and Y. Yamada, Phys. Rev. D 62, 055006 (2000).

[52] G. Degrassi, S. Heinemeyer, W. Hollik, P. Slavich, and G. Weiglein, Eur. Phys. J. C 28, 133 (2003).

[53] S. Heinemeyer, W. Hollik, and G. Weiglein, Phys. Rep. 425, 265 (2006).

[54] B. Allanach, A. Djouadi, J. Kneur, W. Porod, and P. Slavich, J. High Energy Phys. 09 (2004) 044.

[55] S. Heinemeyer, W. Hollik, and G. Weiglein, Comput. Phys. Commun. 124, 76 (2000); T. Hahn, S. Heinemeyer, W. Hollik, H. Rzehak, and G. Weiglein, Comput. Phys. Commun. 180, 1426 (2009); see http://www.feynhiggs.de.

[56] J. Lee, A. Pilaftsis, M. Carena, S. Y. Choi, M. Drees, J. Ellis, and C. E. M. Wagner, Comput. Phys. Commun. 156, 283 (2004); J. Lee, M. Carena, J. Ellis, A. Pilaftsis, and C. Wagner, Comput. Phys. Commun. 180, 312 (2009); 184, 1220 (2013).

[57] S. Martin, Phys. Rev. D 65, 116003 (2002); 66, 096001 (2002); 67, 095012 (2003); 68, 075002 (2003); 70, 016005 (2004); 71, 016012 (2005); 71, 116004 (2005); 75, 055005 
(2007); S. Martin and D. Robertson, Comput. Phys. Commun. 174, 133 (2006).

[58] R. V. Harlander, P. Kant, L. Mihaila, and M. Steinhauser, Phys. Rev. Lett. 100, 191602 (2008); 101, 039901(E) (2008); P. Kant, R. V. Harlander, L. Mihaila, and M. Steinhauser, J. High Energy Phys. 08 (2010) 104.

[59] J. Gunion and A. Turski, Phys. Rev. D 39, 2701 (1989); 40, 2333 (1989).

[60] A. Brignole, J. Ellis, G. Ridolfi, and F. Zwirner, Phys. Lett. B 271, 123 (1991).

[61] A. Brignole, Phys. Lett. B 277, 313 (1992).

[62] M. Diaz and H. Haber, Phys. Rev. D 45, 4246 (1992); M. Diaz, Ph.D. thesis, University of California, Santa Cruz, 1992, SCIPP-92/13.

[63] P. H. Chankowski, S. Pokorski, and J. Rosiek, Phys. Lett. B 274, 191 (1992).

[64] M. Frank, Ph.D. thesis, University of Karlsruhe, 2002.

[65] J. Küblbeck, M. Böhm, and A. Denner, Comput. Phys. Commun. 60, 165 (1990); T. Hahn, Comput. Phys. Commun. 140, 418 (2001); T. Hahn and C. Schappacher, Comput. Phys. Commun. 143, 54 (2002). The program and the user's guide are available via http://www.feynarts.de.

[66] T. Hahn and M. Pérez-Victoria, Comput. Phys. Commun. 118, 153 (1999).

[67] F. del Aguila, A. Culatti, R. Munoz Tapia, and M. PerezVictoria, Nucl. Phys. B537, 561 (1999).

[68] W. Siegel, Phys. Lett. 84B, 193 (1979); D. Capper, D. Jones, and P. van Nieuwenhuizen, Nucl. Phys. B167, 479 (1980).

[69] D. Stöckinger, J. High Energy Phys. 03 (2005) 076.

[70] W. Hollik and D. Stöckinger, Phys. Lett. B 634, 63 (2006).

[71] G. Weiglein, R. Scharf, and M. Böhm, Nucl. Phys. B416, 606 (1994); G. Weiglein, R. Mertig, R. Scharf, and M. Böhm, in New Computing Techniques in Physics
Research 2, edited by D. Perret-Gallix (World Scientific, Singapore, 1992), p. 617.

[72] W. Hollik and H. Rzehak, Eur. Phys. J. C 32, 127 (2003).

[73] S. Heinemeyer, W. Hollik, H. Rzehak, and G. Weiglein, Eur. Phys. J. C 39, 465 (2005).

[74] L. Hofer, U. Nierste, and D. Scherer, J. High Energy Phys. 10 (2009) 081.

[75] D. Noth and M. Spira, Phys. Rev. Lett. 101, 181801 (2008).

[76] A. Denner, Fortschr. Phys. 41, 307 (1993).

[77] A. Akeroyd and S. Baek, Phys. Lett. B 525, 315 (2002).

[78] K. Assamagan and Y. Coadou, Report No. ATL-PHYS2001-017; R. Kinnunen (private communication).

[79] A. Ferrari, in $\mathrm{CH}^{ \pm}$arged 2006, Uppsala, Sweden, 2006 (unpublished); see http://indico.cern.ch/conferenceOther Views.py?view $=$ standard $\&$ confId $=3619$.

[80] E. Boos, V. Bunichev, M. Carena, and C. Wagner, arXiv: hep-ph/0507100.

[81] M. Carena, S. Heinemeyer, C. Wagner, and G. Weiglein, Eur. Phys. J. C 26, 601 (2003).

[82] M. Carena, S. Heinemeyer, C. Wagner, and G. Weiglein, Eur. Phys. J. C 45, 797 (2006).

[83] S. Heinemeyer, W. Hollik, and G. Weiglein, J. High Energy Phys. 06 (2000) 009.

[84] Tevatron Electroweak Working Group, arXiv:1107.5255; see http://tevewwg.fnal.gov/top/.

[85] S. Gennai, S. Heinemeyer, A. Kalinowski, R. Kinnunen, S. Lehti, A. Nikitenko, and G. Weiglein, Eur. Phys. J. C 52, 383 (2007).

[86] J. Coarasa, D. Garcia, J. Guasch, R. Jimenez, and J. Sola, Eur. Phys. J. C 2, 373 (1998); A. Belyaev, D. Garcia, J. Guasch, and J. Sola, Phys. Rev. D 65, 031701 (2002); J. High Energy Phys. 06 (2002) 059; K. A. Assamagan, J. Guasch, S. Moretti, and S. Peñaranda, arXiv:hep-ph/ 0402212; Czech. J. Phys. 55, B787 (2005). 